\title{
MATHER DISCREPANCY AS AN EMBEDDING DIMENSION IN THE SPACE OF ARCS
}

\author{
HUSSEIN MOURTADA, ANA J. REGUERA
}

\begin{abstract}
Let $X$ be a variety over a field $k$ and let $X_{\infty}$ be its space of arcs. We study the embedding dimension of the complete local ring $\widehat{A}:=\widehat{\mathcal{O}_{X_{\infty}, P_{E}}}$ where $P_{E}$ is the stable point defined by a divisorial valuation $\nu_{E}$ on $X$. Assuming char $k=0$, we prove that embdim $\widehat{A}=\widehat{k}_{E}+1$ where $\widehat{k}_{E}$ is the Mather discrepancy of $X$ with respect to $\nu_{E}$. We also obtain that $\operatorname{dim} \widehat{A}$ has as lower bound the MatherJacobian log-discrepancy of $X$ with respect to $\nu_{E}$. For $X$ normal and complete intersection, we prove as a consequence that points $P_{E}$ of codimension one in $X_{\infty}$ have discrepancy $k_{E} \leq 0 .^{1}$
\end{abstract}

\section{INTRODUCTION}

In 1968, J. Nash introduced the space of $\operatorname{arcs} X_{\infty}$ of an algebraic variety $X$ in order to study the singularities of $X$. More precisely, he wanted to understand what the various resolutions of singularities have in common; his work being established just after the proof of resolution of singularities in characteristic zero by H. Hironaka. Nash's work was spread by H. Hironaka and later by M. Lejeune-Jalabert.

The development of motivic integration gave powerful tools for studying finiteness properties in the (not of finite type) $k$-scheme $X_{\infty}$. Two main ideas in J. Denef and F. Loeser's article [DL] appear in this work: the change of variables formula in motivic integration and the stability property, which had already appeared in Kolchin's work on differential algebra. More precisely, based on this stability property, in [Re1] and [Re2] (see also [Re3]) we introduced stable points of $X_{\infty}$, which are certain fat points of finite codimension in $X_{\infty}$. We proved that, if $P$ is stable then the complete local ring $\widehat{\mathcal{O}_{X_{\infty}, P}}$ is a Noetherian ring. From this result we proved a Curve Selection Lemma ending at stable points of $X_{\infty}$. Stable points form a natural framework whenever induced morphisms $\eta_{\infty}: Y_{\infty} \rightarrow X_{\infty}$ are consider, where $\eta: Y \rightarrow X$ is of finite type and locally dominant ([Re2] and [Re3]).

Mori theory is also related to the study of the space of arcs. The recent work of T. de Fernex and R. Docampo [dFD] (see also [dF2]) has confirmed this relationship. In fact, a divisorial valuation $\nu=\nu_{E}$ on $X$ defines a stable point $P_{E}$ on $X_{\infty}$ and, assuming the existence of a resolution of singularities and applying the previous Curve Selection Lemma, we can characterize $\operatorname{dim} \mathcal{O}_{X_{\infty}, P_{E}}=1$ in terms of a property of lifting wedges centered at $P_{E}([\operatorname{Re} 3])$. Then, de Fernex and Docampo's result, which gives an approach to Nash's project, can be understood as follows: assuming char $k=0$, we have that if $\nu_{E}$ is a terminal valuation then $\operatorname{dim} \mathcal{O}_{X_{\infty}, P_{E}}=\operatorname{dim} \widehat{\mathcal{O}_{X_{\infty}, P_{E}}}=1$. On the other hand, several examples of a normal hypersurface $X$ and an essential valuation $\nu_{E}$ for which the property of lifting wedges centered at $P_{E}$ does not hold have been studied ([IK], [dF1], [JK]). One of

\footnotetext{
${ }^{1}$ Keywords: Space of arcs, embedding dimension, Mather discrepancy. MSC: 13A18, 13H99, 14B05, 14E15, 14J17.
} 
the key points in producing such examples is to require $k_{E} \geq 1$ where $k_{E}$ is the discrepancy of $X$ with respect to $E$. This suggests a connection between $\operatorname{dim} \mathcal{O}_{X_{\infty}, P_{E}}$, or $\operatorname{dim} \widehat{\mathcal{O}_{X_{\infty}, P_{E}}}$, and geometric invariants of $\left(X, \nu_{E}\right)$.

Understanding the algebraic properties of the rings $\widehat{\mathcal{O}_{X_{\infty}, P}}$ or of $\mathcal{O}_{X_{\infty}, P}, P$ being stable, is an important problem; it leads towards the study nonconstant families of arcs in $X_{\infty}$. In particular, one of our main goals is to compute $\operatorname{dim} \mathcal{O}_{X_{\infty}, P}$. In general, for any stable point $P$, an upper bound on the dimension of $\mathcal{O}_{X_{\infty}, P}$ follows from the stability property: Expressed in terms of cylinders, stable points are precisely the generic points of the irreducible cylinders in $X_{\infty}$ and $\operatorname{dim} \mathcal{O}_{X_{\infty}, P}$ is bounded from above by the codimension as cylinder of the closure of $P$ in $X_{\infty}$ (see (4) in 2.3). If $X$ is nonsingular at the center of $P$ in $X$, then the $\operatorname{ring} \mathcal{O}_{X_{\infty}, P}$ is regular and the dimension is equal to its upper bound, but in general the inequality in the bound is strict. From the change of variables formula in motivic integration it follows that the codimension as cylinder of the closure $N_{E}$ of $P_{E}$ is equal to $\widehat{k}_{E}+1$ where $\widehat{k}_{E}$ is the Mather discrepancy of $X$ with respect to $E$, introduced in [dFI] (see also $[\mathrm{I}]$ ). Hence $\operatorname{dim} \mathcal{O}_{X_{\infty}, P_{E}} \leq \widehat{k}_{E}+1$.

In this article we study the embedding dimension of $\mathcal{O}_{\left(X_{\infty}\right)_{\text {red }}, P_{E}}$. We prove that, assuming char $k=0$, we have

$$
\operatorname{embdim} \widehat{\mathcal{O}_{X_{\infty}, P_{E}}}=\operatorname{embdim} \mathcal{O}_{\left(X_{\infty}\right)_{\text {red }}, P_{E}}=\widehat{k}_{E}+1
$$

that is, the embedding dimension of $\mathcal{O}_{\left(X_{\infty}\right)_{\text {red }}, P_{E}}$ is equal to the codimension as cylinder of $N_{E}$. Moreover, we describe explicitly a minimal system of coordinates of $\left(X_{\infty}\right)_{\text {red }}$ at $P_{E}$. Applying this, we obtain the following lower bound:

$$
\operatorname{dim} \widehat{\mathcal{O}_{X_{\infty}, P_{E}}} \geq \widehat{k}_{E}-\nu_{E}\left(J a c_{X}\right)+1
$$

where $J a c_{X}$ is the Jacobian ideal of $X$. In particular, if $X$ is normal and complete intersection then $\operatorname{dim} \widehat{\mathcal{O}_{X_{\infty}, P_{E}}} \geq k_{E}+1$. Hence, in this case, $\operatorname{dim} \mathcal{O}_{X_{\infty}, P_{E}}=1$, or $\operatorname{dim} \widehat{\mathcal{O}_{X_{\infty}, P_{E}}}=1$, implies $k_{E} \leq 0$.

The graded algebra associated to the divisorial valuation $\nu_{E}$ plays an essential role in this study. The natural coordinates of $\left(X_{\infty}\right)_{\text {red }}$ at $P_{E}$ are obtained by specialization techniques to the graded algebra of $\nu_{E}$ adapted from B. Teissier ([ZT], $[\mathrm{GT}],[\mathrm{Te}])$. These techniques are applied to a general projection $X \rightarrow \mathbb{A}^{d}$ and the induced valuation on $\mathbb{A}^{d}$. Such coordinates are introduced in [Re4]. In section 3 of this paper we prove that they also provide minimal coordinates of $\left(X_{\infty}\right)$ red at $P_{E}$ and we conclude (1). The way we obtain this proof is, with the language in [Te], embedding $X$ in a complete intersection $X^{\prime}$ which is an overweight deformation of an affine toric variety associated to the divisorial valuation $\nu_{E}$. In section 4 we prove the lower bound for $\operatorname{dim} \widehat{\mathcal{O}_{X_{\infty}, P_{E}}}$ in (2); for this we embed $X$ in a general complete intersection $X^{\prime}$. The important fact used here is that $X$ can be substituted by $X^{\prime}$ in order to compute the local rings $\widehat{\mathcal{O}_{X_{\infty}, P_{E}}}$ ([Re3], cf. 2.3 (ii) and (ix) of this paper). All these results extend to arbitrary stable points $P$ of $X_{\infty}$.

Acknowledgements. We are grateful to Monique Lejeune-Jalabert, for so many enlightening discussions during so many years. We thank O. Piltant for his suggestions and comments. 


\section{Preliminaires}

2.1. In this section we will set the notation and recall some properties of the space of arcs and their stable points. For more details see [DL], [EM], [IK], [Re3].

Let $k$ be a perfect field and let $X$ be a $k$-scheme. Given a field extension $k \subseteq K$, a $K$-arc on $X$ is a $k$-morphism Spec $K[[t]] \rightarrow X$. The $K$-arcs on $X$ are the $K$ rational points of a $k$-scheme $X_{\infty}$ called the space of arcs of $X$. More precisely, $X_{\infty}=\lim _{\leftarrow} X_{n}$, where, for $n \in \mathbb{N}, X_{n}$ is the $k$-scheme of $n$-jets whose $K$-rational points are the $k$-morphisms Spec $K[t] /(t)^{n+1} \rightarrow X$. In fact, the projective limit is a $k$-scheme because the natural morphisms $X_{n^{\prime}} \rightarrow X_{n}$, for $n^{\prime} \geq n$, are affine morphisms. We denote by $j_{n}: X_{\infty} \rightarrow X_{n}, n \geq 0$, the natural projections.

For every $k$-algebra $A$, we have a natural isomorphism

$$
H_{k}\left(\operatorname{Spec} A, X_{\infty}\right) \cong H o m_{k}(\operatorname{Spec} A[[t]], X) \text {. }
$$

Given $P \in X_{\infty}$, with residue field $\kappa(P)$, we denote by $h_{P}:$ Spec $\kappa(P)[[t]] \rightarrow X$ the $\kappa(P)$-arc on $X$ corresponding by $(3)$ to the $\kappa(P)$-rational point of $X_{\infty}$ defined by $P$. The image in $X$ of the closed point of Spec $\kappa(P)[[t]]$, or equivalently, the image $P_{0}$ of $P$ by $j_{0}: X_{\infty} \rightarrow X=X_{0}$ is called the center of $P$. Then, we denote by $\nu_{P}$ the order function $\operatorname{ord}_{t} h_{P}^{\sharp}: \mathcal{O}_{X, P_{0}} \rightarrow \mathbb{N} \cup\{\infty\}$. It also follows from (3) that a $K$-arc on $X_{\infty}$ is equivalent to a $K$-wedge, i.e. a $k$-morphism $\Phi: \operatorname{Spec} K[[\xi, t]] \rightarrow X$.

The space of arcs of the affine space $\mathbb{A}_{k}^{N}=\operatorname{Spec} k\left[x_{1}, \ldots, x_{N}\right]$ is $\left(\mathbb{A}_{k}^{N}\right)_{\infty}=$ Spec $k\left[\underline{X}_{0}, \underline{X}_{1}, \ldots, \underline{X}_{n}, \ldots\right]$ where for $n \geq 0, \underline{X}_{n}=\left(X_{1 ; n}, \ldots, X_{N ; n}\right)$ is an $N$-uple of variables. For any $f \in k\left[x_{1}, \ldots, x_{N}\right]$, let $\sum_{n=0}^{\infty} F_{n} t^{n}$ be the Taylor expansion of $f\left(\sum_{n} \underline{X}_{n} t^{n}\right)$, hence $F_{n} \in k\left[\underline{X}_{0}, \ldots, \underline{X}_{n}\right]$. Equivalently, $\sum_{n=0}^{\infty} F_{n} t^{n}$ is the image of $f$ by the morphism of $k$-algebras $\mathcal{O}_{\mathbb{A}_{k}^{N}} \rightarrow \mathcal{O}_{\left(\mathbb{A}_{k}^{N}\right)_{\infty}}[[t]]$ induced in (3) by the identity map in $\left(\mathbb{A}_{k}^{N}\right)_{\infty}$. If $X \subseteq \mathbb{A}_{k}^{N}$ is affine, and $I_{X} \subset k\left[x_{1}, \ldots, x_{N}\right]$ is the ideal defining $X$ in $\mathbb{A}_{k}^{N}$, then we have

$$
X_{\infty}=\operatorname{Spec} k\left[\underline{X}_{0}, \underline{X}_{1}, \ldots, \underline{X}_{n}, \ldots\right] /\left(\left\{F_{n}\right\}_{n \geq 0, f \in I_{X}}\right) .
$$

Analogously, if $X=$ Spec $k\left[\left[x_{1}, \ldots, x_{N}\right]\right] / I_{X}$ then we have

$$
X_{\infty}=\operatorname{Spec} k\left[\left[\underline{X}_{0}\right]\right]\left[\underline{X}_{1}, \ldots, \underline{X}_{n}, \ldots\right] /\left(\left\{F_{n}\right\}_{n \geq 0, f \in I_{X}}\right) .
$$

2.2. Let $X$ be a separated $k$-scheme which is locally of finite type over some Noetherian complete local ring $R_{0}$ with residue field $k$. Note that $X$ may be a reduced separated $k$-scheme of finite type, and it may also be a $k$-scheme Spec $\widehat{R}$, being $\widehat{R}$ the completion of a local ring $R$ which is a $k$-algebra of finite type. In [Re3] the stable points of $X_{\infty}$ were defined as follows:

First, if $X$ is affine and irreducible and $P$ is a point of $X_{\infty}$, i.e. a prime ideal of $\mathcal{O}_{X_{\infty}}$, then the following conditions are equivalent:

(a) There exist $n_{1} \in \mathbb{N}$, and $G \in \mathcal{O}_{X_{\infty}} \backslash P, G \in \mathcal{O}_{X_{n_{1}}}$ such that, for $n \geq n_{1}$, the map $X_{n+1} \longrightarrow X_{n}$ induces a trivial fibration

$$
\overline{j_{n+1}(Z(P))} \cap\left(X_{n+1}\right)_{G} \longrightarrow \overline{j_{n}(Z(P))} \cap\left(X_{n}\right)_{G}
$$

with fiber $\mathbb{A}_{k}^{d}$, where $d=\operatorname{dim} X,\left(X_{n}\right)_{G}$ is the open subset $X_{n} \backslash Z(G)$ of $X_{n}$ and $\overline{j_{n}(Z(P))}$ is the closure of $j_{n}(Z(P))$ in $X_{n}$ with the reduced structure.

(b) There exists $G \in \mathcal{O}_{X_{\infty}} \backslash P$ such that the ideal $P\left(\mathcal{O}_{X_{\infty}}\right)_{G}$ is the radical of a finitely generated ideal of $\left(\mathcal{O}_{X_{\infty}}\right)_{G}$.

We say that the point $P$ is stable if the previous conditions hold ([Re2] and [Re3], see also J. Denef, F. Loeser [DL], lemma 4.1, and M. Lejeune-Jalabert [Le] for the stability property on the maps $\left.j_{n+1}\left(X_{\infty}\right) \rightarrow j_{n}\left(X_{\infty}\right)\right)$. 
In general, i.e. for $X$ not necessarily irreducible, the set of stable points of $X_{\infty}$ is the union of the sets of stable points of the irreducible components of $X$. Besides this union is disjoint (see (i) in 2.3 bellow).

Recall that a subset $C$ of $X_{\infty}$ is a cylinder if it is of the form $C=j_{n}^{-1}(S)$ for some $n$ and some constructible subset $S \subseteq X_{n}$ ([EM], sec. 5). Hence, from (b) above it follows that the stable points of $X_{\infty}$ are precisely the generic points of the irreducible cylinders.

2.3. The next properties of stable points will be used in the next sections. The first ones, (i) to (iv), are direct consequence of the definition of stable points and of the stability property in [DL], and property (v) applies also well-known facts of the theory of valuations:

([Re3], prop. 3.7) Let $P$ be a stable point of $X_{\infty}$, then the following holds:

(i) Let $X_{0}$ be an irreducible component of $X$ such that $P \in\left(X_{0}\right)_{\infty}$. Then, the arc $h_{P}:$ Spec $\kappa(P)[[t]] \rightarrow X_{0}$ defined by $P$ is a dominant morphism.

(ii) Let $U$ be any irreducible open affine subscheme of $X$ which contains the image of $h_{P}$, then

$$
\mathcal{O}_{X_{\infty}, P}=\mathcal{O}_{U_{\infty}, P}
$$

Moreover, there exits $X^{\prime} \subseteq \mathbb{A}_{k}^{N}$ a complete intersection scheme which contains $U$ and of $\operatorname{dimension} \operatorname{dim} U$ and, for any such $X^{\prime}$, we have that

$$
\mathcal{O}_{\left(X_{\infty}\right)_{\text {red }}, P} \cong \mathcal{O}_{\left(U_{\infty}\right)_{\text {red }}, P} \cong \mathcal{O}_{\left(X_{\infty}^{\prime}\right)_{\text {red }}, P}
$$

where we also denote by $P$ the point induced by $P$ in $\left(X_{\infty}\right)_{\text {red }}$ and in $\left(X_{\infty}^{\prime}\right)_{\text {red }}$. Therefore $X_{\infty}$ is irreducible at $P$, i.e. the nilradical of the ring $\mathcal{O}_{X_{\infty}, P}$ is a prime ideal.

(iii) The residue field $\kappa(P)$ of $P$ on $X_{\infty}$ is a countably pure trascendental extension of a finite extension of $k$. This implies that $\kappa(P)$ is a separably generated field extension of $k$.

(iv) $\operatorname{dim} \mathcal{O}_{\overline{j_{n}\left(X_{\infty}\right)}, P_{n}}$ is constant for $n>>0$, where $\overline{j_{n}\left(X_{\infty}\right)}$ is the closure of $j_{n}\left(X_{\infty}\right)$ in $X_{n}$, with the reduced structure, and $P_{n}$ is the prime ideal $P \cap$ $\mathcal{O}_{\overline{j_{n}}\left(X_{\infty}\right)}$. Since

$$
\operatorname{dim} \mathcal{O}_{X_{\infty}, P} \leq \sup _{n} \operatorname{dim} \mathcal{O}_{\overline{j_{n}\left(X_{\infty}\right)}, P_{n}}
$$

this implies that $\operatorname{dim} \mathcal{O}_{X_{\infty}, P}<\infty$.

(v) Let $\nu_{P}$ be the valuation on the function field $K\left(X_{0}\right)$ of $X_{0}$ defined by the arc $h_{P}, X_{0}$ being the irreducible component of $X$ such that $P \in\left(X_{0}\right)_{\infty}$. Then, either $P_{0}$ is the generic point of $X$ and in this case $\nu_{P}$ is trivial, or $\nu_{P}$ is a divisorial valuation.

Property (i) is equivalent to the statement in [EM] lemma 5.1 for cylinders. In property (iv), the right hand side term in (4) is the definition of the codimension of the cylinder $Z(P)$ (see [EM] sec. 5); but the inequality in (4) may be strict. For property $(\mathrm{v})$ in the setting of cylinders, see $[\mathrm{dFEI}]$ and also [ELM]. The next property compares the local rings at stable points of the space of arcs of $X=$ Spec $R$, where $R$ is a local ring which is a $k$-algebra of finite type, and of of $\widehat{X}=$ Spec $\widehat{R}$, where $\widehat{R}$ is the completion of $R$ :

(vi) Let $P$ be a stable point of $X_{\infty}$, where $X=\operatorname{Spec} R$ as before, whose center in $X$ is the maximal ideal of $R$. Then $P$ induces a stable point in $\widehat{X}_{\infty}$, that we also denote by $P$, and we have

$$
\widehat{\mathcal{O}_{X_{\infty}, P}}=\widehat{\mathcal{O}_{\widehat{X}_{\infty}, P}} .
$$


The following finiteness property of the stable points, which is the main result in [Re2], is expressed in terms of the local ring $\mathcal{O}_{X_{\infty}, P}$, or more precisely, its formal completion. It implies a Curve Selection Lemma in $X_{\infty}$ ending at a stable point $P$ ([Re2], corol. 4.8). Property (viii) below helps to understand this local ring.

Finiteness property of the stable points ([Re2] th. 4.1). Let $P$ be a stable point of $X_{\infty}$, then:

(vii) The formal completion $\widehat{\mathcal{O}_{\left(X_{\infty}\right)_{\text {red }}}, P}$ of the local ring of $\left(X_{\infty}\right)_{\text {red }}$ at a stable point $P$ is a Noetherian ring.

(viii) Moreover, if $X$ is affine, then there exists $G \in \mathcal{O}_{X_{\infty}} \backslash P$ such that the ideal $P\left(\mathcal{O}_{\left(X_{\infty}\right)_{\text {red }}}\right)_{G}$ is a finitely generated ideal of $\left(\mathcal{O}_{\left(X_{\infty}\right)_{\text {red }}}\right)_{G}$.

(ix) ([Re3] th. 3.13 if char $k=0)$ Moreover, we have $\widehat{\mathcal{O}_{X_{\infty}, P}} \cong \widehat{\mathcal{O}_{\left(X_{\infty}\right)_{\text {red }}, P}}$.

From this it follows that, if $P$ is a stable point of $X_{\infty}$, then the maximal ideal of $\widehat{\mathcal{O}_{X_{\infty}, P}}$ is $P \widehat{\mathcal{O}_{X_{\infty}, P}}$, and even more,

$$
\text { embdim } \widehat{\mathcal{O}_{X_{\infty}, P}}=\operatorname{embdim} \mathcal{O}_{\left(X_{\infty}\right)_{\text {red }}, P}
$$

(see [Bo] cap. III, sec. 2, no. 12, corol. 2).

Stable points behave well under birational proper $k$-morphisms and, if we assume that char $k=0$, then also under $k$-morphisms locally of finite type which are locally dominant:

(x) ([Re3] prop. 4.1) Let $\pi: Y \rightarrow X$ be a birational and proper $k$-morphism, then the morphism $\pi_{\infty}: Y_{\infty} \rightarrow X_{\infty}$ induces a one to one map between the stable points of $Y_{\infty}$ and the stable points of $X_{\infty}$. Besides, if $Q$ is a stable point of $Y_{\infty}$ and $P$ its image, then the induced morphism $\widehat{\mathcal{O}_{X_{\infty}, P}} \rightarrow \widehat{\mathcal{O}_{Y_{\infty}, Q}}$ is surjective and induces an isomorphism on the residue fields $\kappa(P) \cong \kappa(Q)$.

(xi) ([Re3] prop. 4.5) Suppose that char $k=0$. Let $\eta: Y \rightarrow X$ be a $k$ morphism locally dominant, then the morphism $\eta_{\infty}: Y_{\infty} \rightarrow X_{\infty}$ induces a map from the set of stable points of $Y_{\infty}$ to the set of stable points of $X_{\infty}$ Besides, if $Q$ is a stable point of $Y_{\infty}$ and $P$ its image by the previous map, then the induced morphism $\left(\mathcal{O}_{X_{\infty}, P}\right)_{\text {red }} \rightarrow\left(\mathcal{O}_{Y_{\infty}, Q}\right)_{\text {red }}$ is an injective local morphism.

Moreover, if $\eta$ is finite and dominant, then $\widehat{\mathcal{O}_{X_{\infty}, P}} \rightarrow \widehat{\mathcal{O}_{Y_{\infty}, Q}}$ is unramified at $Q \widehat{\mathcal{O}_{Y_{\infty}, Q}}$, that is $P \widehat{\mathcal{O}_{Y_{\infty}, Q}}=Q \widehat{\mathcal{O}_{Y_{\infty}, Q}}$, and it indices a finite extension $\kappa(P) \subseteq \kappa(Q)$ on the residue fields.

(xii) ([Re4] prop. 2.5) Let $\eta: Y \rightarrow X$ be an étale $k$-morphism. Then $Y_{\infty}$ is étale over $X_{\infty}$ and, if $Q$ is a stable point of $Y_{\infty}$ and $P$ its image, then $\widehat{\mathcal{O}_{Y_{\infty}, Q}} \cong \widehat{\mathcal{O}_{X_{\infty}, P}} \otimes_{\kappa(P)} \kappa(Q)$.

2.4. Suppose that there exists a resolution of singularities $\pi: Y \rightarrow X$ of $X$, i.e. a proper, birational $k$-morphism, with $Y$ is smooth, such that the induced morphism $Y \backslash \pi^{-1}(\operatorname{Sing} X) \rightarrow X \backslash \operatorname{Sing} X$ is an isomorphism. Let $E$ be a divisor on $Y$ and let $Y_{\infty}^{E}$ be the inverse image of $E$ by the natural projection $j_{0}^{Y}: Y_{\infty} \rightarrow Y$. Then $Y_{\infty}^{E}$ is an irreducible subset of $Y_{\infty}$ whose generic point $P_{E}^{Y}$ is a stable point of $Y_{\infty}$. Besides, the image $P_{E}^{X}$ of $P_{E}^{Y}$ by the morphism $\pi_{\infty}: Y_{\infty} \rightarrow X_{\infty}$ is a stable point of $X_{\infty}$ (see (x) above). We will denote $P_{E}=P_{E}^{X}$ if there is no possible ambiguity. Note that $P_{E}$ only depends on the divisorial valuation $\nu_{E}$ defined by $E$, more precisely, if $\pi^{\prime}: Y^{\prime} \rightarrow X$ is another resolution of singularities such that the center $E^{\prime}$ of $\nu_{E}$ in $Y^{\prime}$ is a divisor, then the stable point $P_{E^{\prime}}$ defined by $E^{\prime}$ coincides with $P_{E}$. Note also that the order function $\nu_{P_{E}}$ is equal to the restriction of the divisorial valuation $\nu_{E}$ to the local ring of $X$ at the generic point of $\pi(E)$. 
The set $Y_{\infty}^{E}$ is also denoted by $\operatorname{Cont}^{1}(E)$. More generally

$$
\operatorname{Cont}^{e}(E):=\left\{Q^{\prime} \in Y_{\infty} / \nu_{Q^{\prime}}\left(I_{E}\right)=e\right\} \text { for every } e \geq 1
$$

where $I_{E}$ is the ideal defining $E$ in an open affine subset of $Y$. We also have that Cont $^{e}(E)$ is an irreducible subset of $Y_{\infty}$ whose generic point $P_{e E}^{Y}$ is a stable point of $Y_{\infty}$, and the image $P_{e E}^{X}$ (also denoted by $P_{e E}$ ) of $P_{e E}^{Y}$ by $\pi_{\infty}$ is a stable point of $X_{\infty}$.

Example 2.5. Note that there are stable points which are not of the type $P_{e E}$ where $\nu_{E}$ is a divisorial valuation on $X$. For instance, let $X=\mathbb{A}^{1}$ and let $P$ be the prime ideal $\left(x_{0}, x_{3}\right)$ of $\mathcal{O}_{X_{\infty}}=k\left[x_{0}, x_{1}, \ldots\right]$. Then $\nu_{P}$ is the divisorial valuation $\nu_{E}$ defined by $\nu_{E}(x)=1$, hence it is the multiplicity in $k[x]$, but $P \neq P_{E}$.

2.6. If $\pi: Y \rightarrow X$ is a resolution of singularities dominating the Nash blowing up of $X$, then the image of the canonical homomorphism $d \pi: \pi^{*}\left(\wedge^{d} \Omega_{X}\right) \rightarrow \wedge^{d} \Omega_{Y}$ is an invertible sheaf. That is, there exists an effective divisor $\widehat{K}_{Y / X}$ with support in the exceptional locus of $\pi$ such that $d \pi\left(\pi^{*}\left(\wedge^{d} \Omega_{X}\right)\right)=\mathcal{O}_{Y}\left(-\widehat{K}_{Y / X}\right) \wedge^{d} \Omega_{Y}$. For any prime divisor $E$ on $Y$, we define the Mather discrepancy to be

$$
\widehat{k}_{E}:=\operatorname{ord}_{E}\left(\widehat{K}_{Y / X}\right) \text {. }
$$

Note that $\widehat{k}_{E} \neq 0$ implies that $E$ is contained in the exceptional locus of $\pi$, and that $\widehat{k}_{E}$ only depends on the divisorial valuation $\nu_{E}$ defined by $E$. We have $\sup _{n} \operatorname{dim} \mathcal{O}_{\overline{j_{n}\left(X_{\infty}\right)},\left(P_{e E}\right)_{n}}=e\left(\widehat{k}_{E}+1\right)([\mathrm{DL}]$, lemma 3.1, [dFEI], theorem 3.9). Hence the inequality (4) states that

$$
\operatorname{dim} \mathcal{O}_{X_{\infty}, P_{e E}} \leq e\left(\widehat{k}_{E}+1\right) .
$$

On the other hand, if $X$ is normal and $\mathbb{Q}$-Gorenstein (for instance $X$ is a normal complete intersection), the discrepancy of $X$ with respect to $E$ is defined to be the coefficient of $E$ in the divisor $K_{Y / X}$ with exceptional support which is linearly equivalent to $K_{Y}-\pi^{*}\left(K_{X}\right)$. If $X$ is nonsingular then $\widehat{k}_{E}=k_{E}$ ([EM], appendix). Moreover, we have:

(xiii) ([Re3] prop. 4.2 and [Re4] corol. 2.9) If $X$ is nonsingular at the center $P_{0}$ of a stable point $P$ of $X_{\infty}$, then $\mathcal{O}_{X_{\infty}, P}$ is a regular ring of dimension $\operatorname{dim} \mathcal{O}_{X_{\infty}, P}=\sup _{n} \operatorname{dim} \mathcal{O}_{\overline{j_{n}\left(X_{\infty}\right)}, P_{n}}$. In particular, taking $P=P_{e E}$, we have $\operatorname{dim} \mathcal{O}_{X_{\infty}, P_{e E}}=e\left(k_{E}+1\right)$.

In theorem 3.8 will prove that, also in the case that $X$ is singular at $P_{0}$, we have that $e\left(\widehat{k}_{E}+1\right)$ is the embedding dimension of $\mathcal{O}_{\left(X_{\infty}\right)_{\text {red }}, P_{e E}}$.

Example 2.7. Let $X$ be an irreducible formal plane curve over a field $k$ of characteristic zero. Let us consider a (primitive) Puiseux parametrization

$$
\begin{aligned}
& x=u^{\beta_{0}} \\
& y=\sum_{\beta_{0} \leq i} \lambda_{i} u^{i}
\end{aligned}
$$

where $\lambda_{i} \in k$ for every $i \geq \beta_{0}$. Set $e_{0}:=\beta_{0}$ and,

$$
\begin{array}{ll}
\beta_{r+1} & :=\min \left\{i / \lambda_{i} \neq 0 \text { and g.c.d. }\left\{\beta_{0}, \ldots, \beta_{r}, i\right\}<e_{r}\right\}, \\
e_{r+1} & :=\text { g.c.d. }\left\{\beta_{0}, \ldots, \beta_{r+1}\right\}
\end{array}
$$

for $1 \leq r \leq g-1$, being $g$ such that $e_{g}=1$. Let $n_{0}=1$ and $n_{r}:=\frac{e_{r-1}}{e_{r}}$ for $1 \leq r \leq g$ and let $\bar{\beta}_{0}=\beta_{0}$ and $\bar{\beta}_{r}, 1 \leq r \leq g+1$, be defined by

$$
\bar{\beta}_{r}-n_{r-1} \bar{\beta}_{r-1}=\beta_{r}-\beta_{r-1}
$$


hence we have

$\bar{\beta}_{r}>n_{r-1} \bar{\beta}_{r-1} \quad$ for $1 \leq r \leq g, \quad$ and $\quad \bar{\beta}_{g+1} \geq n_{g} \bar{\beta}_{g}$;

$n_{r} \bar{\beta}_{r}$ belongs to the semigroup generated by $\bar{\beta}_{0}, \ldots, \bar{\beta}_{r-1}, 1 \leq r \leq g+1$.

Let us consider $q_{0}, q_{1}, \ldots, q_{g} \in k[x, y]$ and $q_{g+1} \in k[[x, y]]$ such that $q_{g+1}$ defines an equation of the branch, i.e. $X=$ Spec $k[[x, y]] /\left(q_{g+1}\right)$, and $q_{1}, \ldots, q_{g}$ are its approximate roots (see [ZT], appendix). More precisely, $q_{0}, \ldots, q_{g+1}$ can be defined as follows:

$$
q_{0}=x \quad q_{1}=y-\sum_{i<\bar{\beta}_{1}} \lambda_{i} q_{0}^{\frac{i}{\beta_{0}}}
$$

with $\operatorname{ord}_{u}\left(q_{1}\right)=\bar{\beta}_{1}$ and, for $1 \leq r \leq g$,

$$
q_{r+1}=q_{r}^{n_{r}}-c_{r} q_{0}^{b_{r, 0}} \ldots q_{r-1}^{b_{r, r-1}}-\sum_{\gamma=\left(\gamma_{0}, \ldots, \gamma_{r}\right)} c_{\gamma} q_{0}^{\gamma_{0}} \ldots q_{r}^{\gamma_{r}}, \quad 1 \leq r \leq g
$$

with $\operatorname{ord}_{u}\left(q_{r+1}\right)=\bar{\beta}_{r+1}($ resp. $\infty)$ for $1 \leq r<g($ resp. $r=g)$, where $\left\{b_{r, i}\right\}_{i=0}^{r-1}$ are the unique nonnegative numbers satisfying $b_{r, i}<n_{i}$ for $1 \leq i \leq r-1$ and $n_{r} \bar{\beta}_{r}=\sum_{0 \leq i<r} b_{r, i} \bar{\beta}_{i}$, for each sequence $\gamma$ of nonnegative integers in the right hand side we have $n_{r} \bar{\beta}_{r}<\sum_{i=0}^{r} \gamma_{i} \bar{\beta}_{i}<\bar{\beta}_{r+1}\left(\right.$ resp. $\left.n_{r} \bar{\beta}_{r}<\sum_{i=0}^{r} \gamma_{i} \bar{\beta}_{i}\right)$ if $1 \leq r<g$ (resp. if $r=g+1$ ) and $c_{r}, c_{\gamma} \in k$ and $c_{r} \neq 0$. For more details on approximate roots and the space of arcs of a plane branch see [Mo] and [LMR].

Let $\nu=\nu_{E}$ be the divisorial valuation on $X$ given by $\operatorname{ord}_{u}$, and let $P=P_{E}$ be the stable point in $X_{\infty}$ defined by $\nu$ as in 2.4. Considering the projection $\eta: X \rightarrow \mathbb{A}_{k}^{1}$, $(x, y) \mapsto x$, and applying prop. 4.5 in [Re3] ((xi) in 2.3) we conclude that

$$
P \widehat{\mathcal{O}_{X_{\infty}, P}}=\left(X_{0}, \ldots, X_{\beta_{0}-1}\right) \widehat{\mathcal{O}_{X_{\infty}, P}} .
$$

We will next describe the ring $\widehat{\mathcal{O}_{X_{\infty}, P}}$, and we will see that embdim $\widehat{\mathcal{O}_{X_{\infty}, P}}=\beta_{0}$, which is equal to the multiplicity of $X$ (see [Re3], corol. 5.7).

First note that $P \mathcal{O}_{X_{\infty}, P}$ is generated by $\mathcal{Q}:=\left\{Q_{r ; n}\right\}_{0 \leq r \leq g, n_{r-1} \bar{\beta}_{r-1} \leq n<\bar{\beta}_{r}}$, even more, there exists $G \in \mathcal{O}_{X_{\infty}} \backslash P$ such that $P\left(\mathcal{O}_{X_{\infty}}\right)_{G}=(\mathcal{Q})\left(\mathcal{O}_{X_{\infty}}\right)_{G}$ (we may take $\left.G:=\prod_{0 \leq r \leq g} Q_{r ; \bar{\beta}_{r}}\right)$. More precisely, $(\mathcal{Q})$ defines a prime ideal in $\left(\mathcal{O}_{\left(\mathbb{A}^{2}\right)_{\infty}}\right)_{G}$ (see [Re4], prop 4.5) whose extension to $\left(\mathcal{O}_{X_{\infty}}\right)_{G}$ is $P\left(\mathcal{O}_{X_{\infty}}\right)_{G}$. Note that, setting $f:=q_{g+1} \in k[[x, y]]$, the following holds:

(i) $\nu(\operatorname{Jac}(f))=\nu\left(\frac{\partial f}{\partial y}\right)=n_{g} \bar{\beta}_{g}-\beta_{g}$. Set $\epsilon:=n_{g} \bar{\beta}_{g}-\beta_{g}$,

(ii) for all $n \geq 0$, the class of $\frac{\partial F_{\epsilon+n}}{\partial Y_{n}}$ in $\mathcal{O}_{X_{\infty}, P}$ is a unit and, for $n^{\prime}>n$, the class of $\frac{\partial F_{\epsilon+n}}{\partial Y_{n^{\prime}}}$ in $\mathcal{O}_{X_{\infty}, P}$ belongs to $P \mathcal{O}_{X_{\infty}, P}$.

(iii) $F_{0}, \ldots, F_{\epsilon-1}$ belong to $(\mathcal{Q})^{2} \mathcal{O}_{\left(\mathbb{A}_{k}^{2}\right)_{\infty}}$.

From this it follows that

$$
\kappa(P) \cong k\left(X_{\beta_{0}+1}, \ldots, X_{n}, \ldots\right)\left[\left\{W_{r}\right\}_{r=0}^{g}\right] /\left(\left\{W_{r}^{n_{r}}-c_{r} W_{0}^{b_{r, 0}} \cdots W_{r-1}^{b_{r, r-1}}\right\}_{r=1}^{g}\right)
$$

where $W_{r}$ is the class of $Q_{r ; \bar{\beta}_{r}}$. We consider the embedding $\kappa(P) \hookrightarrow \widehat{\mathcal{O}_{X_{\infty}, P}}$ which sends $X_{n}, n \geq \beta_{0}$, (resp. $\left.W_{0}\right)$ to $X_{n} \in \widehat{\mathcal{O}_{X_{\infty}, P}}$ (resp. $X_{\beta_{0}} \in \widehat{\mathcal{O}_{X_{\infty}, P}}$ ) and recursively, for $1 \leq r \leq g$, sends $W_{r}$ to a $n_{r}$-root of the image in $\widetilde{\mathcal{O}_{X_{\infty}, P}}$ of $c_{r} W_{0}^{b_{r, 0}} \cdots W_{r-1}^{b_{r, r-1}}$, that exists by Hensel's lemma. In particular, for each $n \geq 0$ we have defined $Y_{n}^{(0)} \in \kappa(P)$ such that $Y_{n}-Y_{n}^{(0)} \in(\mathcal{Q})$. Arguing recursively on $m \geq 1$ and $n \geq 0$, with the lexicographic order on $(m, n)$, from $\left\{F_{\epsilon+n}\right\}_{n \geq 0}$, applying (ii) and Hensel's lemma, and reasoning as in corol. 5.6 in [Re3] it follows that, for $m, n \geq 0$, there exists $Y_{n}^{(m)} \in \kappa(P)\left[X_{0}, \ldots, X_{\beta_{0}-1}\right]$ such that,

$$
F_{\epsilon+n} \equiv L_{\epsilon}\left(Y_{n}-Y_{n}^{(m)}\right) \quad \bmod (\mathcal{Q})^{m}
$$


in the ring $\mathcal{O}_{\left(\mathbb{A}^{2}\right)_{\infty},(\mathcal{Q})}$, where $l:=\frac{\partial f}{\partial y}$, hence $L_{\epsilon}$ is a unit. Therefore, the previous equalities define series $\widetilde{Y}_{n} \in \kappa(P)\left[\left[X_{0}, \ldots, X_{\beta_{0}-1}\right]\right], n \geq 0$, and we conclude that

$$
\widehat{\mathcal{O}_{X_{\infty}, P}} \cong \kappa(P)\left[\left[X_{0}, \ldots, X_{\beta_{0}-1}\right]\right] /\left(\left\{\widetilde{F}_{n}\right\}_{0 \leq n \leq \epsilon-1}\right)
$$

where, for $0 \leq n \leq \epsilon-1, \widetilde{F}_{n}$ is obtained from $F_{n}$ by substituting $Y_{n^{\prime}}$ by $\widetilde{Y}_{n}$, $0 \leq n^{\prime} \leq n$. Since, for $0 \leq r \leq g, n_{r-1} \bar{\beta}_{r-1} \leq n<\bar{\beta}_{r}$, the series obtained from $Q_{n}$ by substituting $Y_{n^{\prime}}$ by $\widetilde{Y}_{n}, 0 \leq n^{\prime} \leq n$, belongs to $\left(X_{0}, \ldots, X_{\beta_{0}-1}\right)$, from (iii) it follows that $\widetilde{F}_{n} \in\left(X_{0}, \ldots, X_{\beta_{0}-1}\right)^{2}$ for $0 \leq n \leq \epsilon-1$. Therefore embdim $\widetilde{\mathcal{O}_{X_{\infty}, P}}=\beta_{0}$.

Remark 2.8. Let $X$ be an algebraic plane curve over a field $k$ of characteristic zero, and suppose that it is analytically irreducible. Then, there exists an étale morphism $X^{\prime} \rightarrow X$ such that the curve $X^{\prime}$ has a Puiseux parametrization

$$
\begin{aligned}
& x^{\prime}=\left(u^{\prime}\right)^{\beta_{0}} \\
& y^{\prime}=\sum_{\beta_{0} \leq i \leq m} \lambda_{i}^{\prime}\left(u^{\prime}\right)^{i}
\end{aligned}
$$

where $\lambda_{i}^{\prime} \in k$ for $\beta_{0} \leq i \leq m$, i.e. the image of $y^{\prime}$ has a finite number of terms. Equivalently, the element $q_{g+1}^{\prime}$ obtained as in (7) from the previous parametrization, which defines an equation of the curve $X^{\prime}$, is a polynomial.

In fact, consider a Puiseux parametrization $x=u^{\beta_{0}}, y=\sum_{\beta_{0} \leq i} \lambda_{i} u^{i}$ of $X$ and keep the notation in example 2.7. Note that the series $\sum_{\beta_{0} \leq i} \lambda_{i} u^{i}$ belongs to the hensalization $k<u>$ of $k[u]_{(u)}$ and also that the element $q_{g+1}$ in (7) belongs to $k\langle x, y\rangle$. Since $X$ is analytically irreducible, there exists $\gamma \in k\langle x, y\rangle, \gamma$ a unit, such that $\gamma q_{g+1}$ is a polynomial in $k[x, y]$. Then taking $x^{\prime}=(\gamma)^{\frac{1}{\bar{\beta}_{1}}} x, y^{\prime}=(\gamma)^{\frac{1}{\bar{\beta}_{0}}} y$ and $u^{\prime}=(\gamma)^{\frac{1}{n_{1} \bar{\beta}_{1}}} u$, we obtain (8). Recall that $n_{1} \bar{\beta}_{1}$ is the least common multiple of $\bar{\beta}_{0}$ and $\bar{\beta}_{1}$. Since char $k=0$, adding a $n_{1} \bar{\beta}_{1}$-root of $\gamma$ defines an étale morphism $X^{\prime} \rightarrow X$.

Example 2.9. Let $X \subset \mathbb{A}_{k}^{5}$ be the hypersurface singularity in [IK], defined by $x_{1}^{3}+x_{2}^{3}+x_{3}^{3}+x_{4}^{3}+x_{5}^{6}=0$ over a field of characteristic $\neq 2,3$. The blowing up $X^{\prime}$ of $X$ at the origin has a unique singular point, and its exceptional locus $E_{\beta}$ is irreducible and defines an essential valuation $\nu_{\beta}$ (i.e. the center of $\nu_{\beta}$ on any resolution of singularities $p: \widetilde{X} \rightarrow X$ is an irreducible component of the exceptional locus of $p$ ). The blowing up $Y$ of $X^{\prime}$ at its singular point is nonsingular, and its exceptional locus is irreducible and defines an essential valuation $\nu_{\alpha}, \nu_{\alpha} \neq \nu_{\beta}$. Let $\pi: Y \rightarrow X$ be the induced resolution of singularities. Let $P_{\alpha}, P_{\beta}$ be the stable points of $X_{\infty}$ defined by $\nu_{\alpha}$ and $\nu_{\beta}$ respectively, and set $N_{\alpha}:=\overline{\left\{P_{\alpha}\right\}}, N_{\beta}:=\overline{\left\{P_{\beta}\right\}}$ and $X_{\infty}^{\text {Sing }}$ the inverse image of Sing $X$ by $j_{0}: X_{\infty} \rightarrow X$. We have $N_{\alpha} \subset N_{\beta}=X_{\infty}^{\text {Sing }}$ ([IK], theorem 4.3).

Let $\Pi: \widetilde{\mathcal{Z}} \rightarrow \mathbb{A}_{k}^{5}$ be the embedded resolution of singularities of $X$ whose restriction to $X$ is $\pi$. There exists a divisor $\widetilde{E}$ on $\widetilde{\mathcal{Z}}$ whose intersection with $Y$ is $E_{\beta}$. Note that $b_{\widetilde{E}}:=\operatorname{ord}_{\widetilde{E}} K_{\widetilde{\mathcal{Z}} / \mathbb{A}^{5}}$ is equal to 4 and $a_{\widetilde{E}}:=\operatorname{ord}_{\widetilde{E}} \Pi^{*}(X)$ is equal to 3 . Since, by the adjunction formula, $k_{E_{\beta}}=b_{\widetilde{E}}-a_{\widetilde{E}}$, we have $k_{E_{\beta}}=1$. Hence, $\widehat{k}_{E_{\beta}}=k_{E_{\beta}}+\nu_{\beta}\left(J_{a c_{X}}\right)=1+2=3$ (see [EM], remark 9.6).

On the other hand, we have

$$
P_{\beta}\left(\mathcal{O}_{X_{\infty}}\right)_{X_{1 ; 1}}=\left(X_{1 ; 0}, X_{2 ; 0}, X_{3 ; 0}, X_{4 ; 0}, X_{5 ; 0}\right)\left(\mathcal{O}_{X_{\infty}}\right)_{X_{1 ; 1}} .
$$

In fact, $\left(X_{1 ; 0}, \ldots, X_{5 ; 0}\right)$ is the prime ideal in $\mathcal{O}_{\left(\mathbb{A}^{5}\right)_{\infty}}$ defined by $\nu_{\widetilde{E}}$, hence its minimal number of generators is $b_{\widetilde{E}}+1=5$ (see (xiii) in 2.6). Besides, the ring $\widehat{\mathcal{O}}_{X_{\infty}, P_{\beta}}$ 
has been described in [Re3] remark 5.16 as follows:

$$
\widehat{\mathcal{O}}_{X_{\infty}, P_{\beta}} \cong \kappa\left(P_{\beta}\right)\left[\left[X_{1 ; 0}, X_{2 ; 0}, X_{3 ; 0}, X_{4 ; 0}, X_{5 ; 0}\right]\right] /\left(\widetilde{F}_{0}, \widetilde{F}_{1}, \widetilde{F}_{2}\right)
$$

where, being $f=x_{1}^{3}+x_{2}^{3}+x_{3}^{3}+x_{4}^{3}+x_{5}^{6}$ and being $\bar{F}_{n}$ the class of $F_{n}$ modulo $\left(X_{1 ; 0}, \ldots, X_{5 ; 0}\right)$, we have that $3=a_{\widetilde{E}}$ is the minimal $n$ such that $\bar{F}_{n} \neq 0$, in fact $\bar{F}_{3}=X_{1 ; 1}^{3}+X_{1 ; 2}^{3}+X_{1 ; 3}^{3}+X_{1 ; 4}^{3}$ and

$$
\kappa\left(P_{\beta}\right) \cong k\left(\left\{X_{i ; 1}, \ldots X_{i ; n}, \ldots\right\}_{2 \leq i \leq 4}\right)\left[X_{1 ; 1}\right] /\left(\bar{F}_{3}\right) .
$$

Besides we have $\widetilde{F}_{0}, \widetilde{F}_{1} \in\left(X_{1 ; 0}, \ldots, X_{5 ; 0}\right)^{2}$ and the initial form $\operatorname{in}\left(\widetilde{F}_{2}\right)$ of $\widetilde{F}_{2}$ in $\kappa\left(P_{\beta}\right)\left[\left[X_{1 ; 0}, \ldots, X_{5 ; 0}\right]\right]$ is $3 \bar{X}_{1 ; 1}^{2} X_{1 ; 0}+3 X_{2 ; 1}^{2} X_{2 ; 0}+3 X_{3 ; 1}^{2} X_{3 ; 0}+3 X_{4 ; 1}^{2} X_{4 ; 0}$ where $\bar{X}_{1 ; 1}$ is the class of $X_{1 ; 1}$ in $\kappa\left(P_{\beta}\right)$. Note that $\nu_{\beta}\left(J a c_{X}\right)=2$, even more, for $1 \leq i \leq 4$, if $f^{i}:=\frac{\partial f}{\partial x_{i}}$ then $\nu_{\beta}\left(f^{i}\right)=2$, i.e. $F_{0}^{i}, F_{1}^{i} \in P_{\beta}, F_{2}^{i} \notin P_{\beta}$, and the coefficient in $\bar{X}_{i ; 0}$ of $\operatorname{in}\left(\widetilde{F}_{2}\right)$ is the class of $F_{2}^{i}$ in $\kappa\left(P_{\beta}\right)$. From this it follows that

$\operatorname{embdim} \widehat{\mathcal{O}_{X_{\infty}, P_{\beta}}}=b_{\widetilde{E}}+1-\left(a_{\widetilde{E}}-\nu_{\beta}\left(J a c_{X}\right)\right)=k_{E_{\beta}}+1+\nu_{\beta}\left(J a c_{X}\right)=\widehat{k}_{E_{\beta}}+1$

which equals 4 . Moreover, in this case

$$
\operatorname{dim} \widehat{\mathcal{O}_{X_{\infty}, P_{\beta}}}=b_{\widetilde{E}}+1-a_{\widetilde{E}}=k_{E_{\beta}}+1=2 .
$$

The argument to compute embdim $\widehat{\mathcal{O}_{X_{\infty}, P_{\beta}}}$ showed in example 2.9 can be generalized to monomial valuations restricted to a normal hyperssurface over a perfect field of any characteristic. But, although, given a variety $X$ and a divisorial valuation $\nu_{E}$, there always exists a complete intersection $X^{\prime}$ containig $X$ of the same dimension and we have $\widehat{\mathcal{O}_{X_{\infty}, P_{E}}} \cong \widehat{\mathcal{O}_{X_{\infty}^{\prime}, P_{E}}}$ (see (ii) and (ix) in 2.3), $X^{\prime}$ is not normal in general. So, there is no hope to extend the result embdim $\widehat{\mathcal{O}_{X_{\infty}, P_{E}}}=\widehat{k}_{E}+1$ applying this argument. For $\operatorname{dim} \widehat{\mathcal{O}_{X_{\infty}, P_{E}}}$, even if $X$ is a normal hypersurface it is not true in general that $\operatorname{dim} \widehat{\mathcal{O}_{X_{\infty}, P_{E}}}$ equals $k_{E}+1$, but we will show that $\operatorname{dim} \widehat{\mathcal{O}_{X_{\infty}, P_{E}}} \geq k_{E}+1$.

\section{Defining minimal coordinates at stable points of the space of arCs}

Let $X$ be a (singular) reduced separated scheme of finite type over a field $k$ of characteristic zero. Let $\nu$ be a divisorial valuation on an irreducible component $X_{0}$ of $X$ whose center lies in Sing $X$ and let $e \in \mathbb{N}$.

Let us consider the stable point $P_{e E}$ of $X_{\infty}$ defined by $\nu$ and $e$, i.e. we consider any resolution of singularities $\pi: Y \rightarrow X$ such that the center of $\nu$ on $Y$ is a divisor $E$, and define $P_{e E}=P_{e E}^{X}$ to be the image by $\pi_{\infty}$ of the generic point $P_{e E}^{Y}$ of $Y_{\infty}^{E}$ (see 2.4). In order to study the ring $\mathcal{O}_{X_{\infty}, P_{e E}}$, or its completion $\widehat{\mathcal{O}_{X_{\infty}, P_{e E}}}$, we may suppose that $X$ is affine, let $X \subseteq \mathbb{A}_{k}^{N}=\operatorname{Spec} k\left[y_{1}, \ldots, y_{N}\right]$. We may also suppose that $\pi: Y \rightarrow X$ dominates the Nash blowing up of $X$ and that, if $x_{i}$ denotes the class of $y_{i}$ in $\mathcal{O}_{X}, 1 \leq i \leq N$, then, after reordering the $x_{i}$ 's, we have

$$
\operatorname{ord}_{E} \pi^{*}\left(d x_{1} \wedge \ldots \wedge d x_{d}\right)=\widehat{k}_{E} \text {. }
$$

where $d=\operatorname{dim} X_{0}$.

Let $\rho: X \rightarrow \mathbb{A}_{k}^{d}$ be the projection on the first $d$ coordinates, let $\eta: Y \rightarrow \mathbb{A}^{d}$ be the composition $\eta=\rho \circ \pi$ and let $P_{e E}^{\mathbb{A}^{d}}$ be the image of $P_{e E}^{Y}$ by $\eta_{\infty}$. Then the discrepancy $k_{E}\left(\mathbb{A}_{k}^{d}\right)$ of $\mathbb{A}_{k}^{d}$ with respect to the valuation induced by $\nu_{E}$ is equal to $\widehat{k}_{E}$ by $(9)$. Besides, we know that the local ring $\mathcal{O}_{\left(\mathbb{A}^{d}\right)_{\infty}, P_{e E}^{\mathbb{A}^{d}}}$ is a regular ring of dimension $e\left(k_{E}\left(\mathbb{A}_{k}^{d}\right)+1\right.$ ) (see (xiii) in 2.6). From this, and applying [Re3], prop. 4.5 
(see (xi) in 2.3) it follows that, if $\mathcal{Q}$ is a regular system of parameters of $\mathcal{O}_{\left(\mathbb{A}^{d}\right)_{\infty}, P_{e E}^{\mathbb{A} d}}$ (hence $\sharp \mathcal{Q}=e\left(\widehat{k}_{E}+1\right)$ ) then we have

(10) $P_{e E} \widehat{\mathcal{O}_{X_{\infty}, P_{e E}}}=(\mathcal{Q}) \widehat{\mathcal{O}_{X_{\infty}, P_{e E}}}$ and $P_{e E} \mathcal{O}_{\left(X_{\infty}\right)_{\mathrm{red}}, P_{e E}}=(\mathcal{Q}) \mathcal{O}_{\left(X_{\infty}\right)_{\mathrm{red}}, P_{e E}}$ in fact, the last assertion follows from the first one by Nakakama's lemma. Therefore embdim $\mathcal{O}_{\left(X_{\infty}\right)_{\text {red }}, P_{e E}}=\operatorname{embdim} \widehat{\mathcal{O}_{X_{\infty}, P_{e E}}} \leq e\left(\widehat{k}_{E}+1\right)([\operatorname{Re} 4]$, corol. 4.10).

Remark 3.1. The previous reasoning does not assure an analogous statement to (10) for $P_{e E}^{X} \mathcal{O}_{X_{\infty}, P_{e E}^{X}}$ since, in general the $P_{e E}^{X}$-adic topology on $\mathcal{O}_{X_{\infty}, P_{e E}^{X}}$ is not separated (see [Re3] example 3.16 and theorem 3.13).

3.2. Moreover, in $[\operatorname{Re} 4]$ we have described a regular system of parameters $\mathcal{Q}$

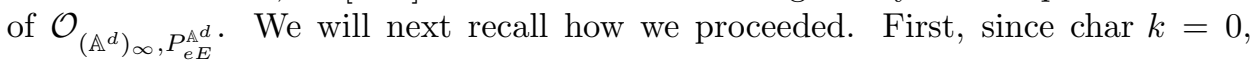
there exists an open subset $U$ of $Y$ with nonempty intersection with $E$, an étale morphism $\widetilde{U} \rightarrow U$ and $\left\{u_{1}, \ldots, u_{d}\right\} \subset \mathcal{O}_{\widetilde{U}},\left\{x_{1}, \ldots, x_{d}\right\} \subset \mathcal{O}_{V}$, where $V$ is an open subset of $X$, such that the following holds: for all closed points $y_{0}$ in an open subset of the strict transform $\widetilde{E}$ of $E$ in $\widetilde{U}$, after a possible replacement of $u_{i}$ by $u_{i}+c_{i}$, $c_{i} \in k, 2 \leq i \leq d$, we may suppose that $\left\{u_{1}, \ldots, u_{d}\right\}$ and $\left\{x_{1}, \ldots, x_{d}\right\}$ are regular systems of parameters in $y_{0}$ and in $\eta \circ \varphi\left(y_{0}\right)$, and besides, the local morphism $\eta^{\sharp}: \mathcal{O}_{V, \eta\left(y_{0}\right)} \rightarrow \mathcal{O}_{\widetilde{U}, y_{0}}$ is given by

$$
\begin{array}{ll}
x_{1} & \mapsto u_{1}^{m_{1}} \\
x_{2} & \mapsto \sum_{1 \leq i \leq m_{2}} \lambda_{2, i} u_{1}^{i}+u_{1}^{m_{2}} u_{2} \\
x_{3} & \mapsto \sum_{1 \leq i \leq m_{3}} \lambda_{3, i}\left(u_{2}\right) u_{1}^{i}+u_{1}^{m_{3}} u_{3} \\
\ldots & \cdots \ldots \\
x_{\delta} & \mapsto \sum_{1 \leq i \leq m_{\delta}} \lambda_{\delta, i}\left(u_{2}, \ldots, u_{\delta-1}\right) u_{1}^{i}+u_{1}^{m_{\delta}} u_{\delta} \\
x_{\delta+1} & \mapsto u_{\delta+1} \\
\ldots & \cdots \cdots \\
x_{d} & \mapsto u_{d}
\end{array}
$$

where $\delta=\operatorname{codim}_{\mathbb{A}^{d}} \overline{\eta\left(\xi_{E}\right)}, \quad m_{1} \leq \operatorname{ord}_{u_{1}} x_{j}, 2 \leq j \leq d, \quad 0<m_{1} \leq m_{2} \leq \ldots \leq m_{d}$, and, for $2 \leq j \leq \delta$ and $0 \leq i \leq m_{j}, \lambda_{j, i}\left(u_{2}, \ldots, u_{j-1}\right)$ belongs to the henselization $k<u_{2}, \ldots, u_{j-1}>$ of the local ring $k\left[u_{2}, \ldots, u_{j-1}\right]_{\left(u_{2}, \ldots u_{j-1}\right)}$, and, if $i<m_{j^{\prime}}$, $j^{\prime}<j$, then $\lambda_{j, i}$ belongs to $k<u_{2}, \ldots, u_{j^{\prime}-1}>$; moreover, with no loss of generality we may also suppose that $\lambda_{j, m_{j}}\left(u_{2}, \ldots, u_{j-1}\right)$ is a unit for $2 \leq j \leq \delta((4)$ in $[\operatorname{Re} 4]$, see also [Re3], proof of prop. 4.5).

3.3. Now, we consider the following situation: Let $j, 2 \leq j \leq d+1$, let $v_{2}, \ldots, v_{j-1}$ so that $u_{1}, v_{2}, \ldots, v_{j-1}, u_{j}, \ldots, u_{d} \in \mathcal{O}_{\widetilde{U}}$ defines a regular system of parameters of $\mathcal{O}_{\widetilde{U}, y_{0}}$ for all closed points $y_{0}$ in an open subset of $\widetilde{E}$ (more precisely, there exist $\left(c_{i}\right)_{i} \in k^{d-1}$ such that $\left(u_{1},\left\{v_{i}+c_{i}\right\}_{i=2}^{\delta},\left\{v_{i}+c_{i}\right\}_{i=\delta+1}^{d}\right)$ is a regular system of parameters of $\left.\mathcal{O}_{\widetilde{U}, y_{0}}\right)$, and let $\theta: \widetilde{U} \rightarrow \operatorname{Spec} k\left[v_{2}, \ldots, v_{j-1}\right]_{h}\left[x_{1}, y\right]$ be the $k$-morphism given by

$$
\begin{aligned}
x_{1} & \mapsto u_{1}^{m_{1}} \\
y & \mapsto \sum_{m_{1} \leq i \leq m} \lambda_{i}\left(v_{2}, \ldots, v_{j-1}\right) u_{1}^{i}+u_{1}^{m} \varrho \bmod \left(u_{1}\right)^{m+1}
\end{aligned}
$$

where $h \in k\left[v_{2}, \ldots, v_{j-1}\right] \backslash\left(v_{2}, \ldots, v_{j-1}\right), m \geq m_{1}, \lambda_{i}\left(v_{2}, \ldots, v_{j-1}\right) \in R_{j-1}:=$ $k<v_{2}, \ldots, v_{j-1}>, \varrho \in \mathcal{O}_{Y, y_{0}}$ and one of the following conditions holds:

(a) $\varrho$ is transcendental over $k\left(u_{1}, v_{2}, \ldots, v_{j-1}\right)$

(b) $\varrho=0$. 
Set $\mathfrak{e}:=$ g.c.d. $\left(\left\{m_{1}\right\} \cup\left\{i / \lambda_{i} \neq 0\right\}\right)$, and define $\beta_{0}:=e_{0}:=m_{1}$, and $\beta_{r+1}:=$ $\min \left\{i / \lambda_{i} \neq 0\right.$ and g.c.d. $\left.\left\{\beta_{0}, \ldots, \beta_{r}, i\right\}<e_{r}\right\}, \quad e_{r+1}:=$ g.c.d. $\left\{\beta_{0}, \ldots, \beta_{r+1}\right\}$ for $1 \leq r<g$, being $g$ such that $e_{g}=\mathfrak{e}$, and $\beta_{g+1}:=m$. Let $n_{r}=\frac{e_{r-1}}{e_{r}}, 1 \leq r \leq g-1$. We define $\left\{\bar{\beta}_{r}\right\}_{r=0}^{g+1}$ from $\left\{\beta_{r}\right\}_{r=0}^{g+1}$ as in (6) in 2.7.

Let $B$ be a domain which is an étale extension of $k\left[v_{2}, \ldots, v_{j-1}\right]_{h}$ and contains $\lambda_{i}\left(v_{2}, \ldots, v_{j-1}\right), m_{1} \leq i \leq m$. Let $\widetilde{\nu}$ be the order function on $B\left[x_{1}, y\right]$ extending $\nu$ and such that $\widetilde{\nu}(\ell)=0$ for all $\ell \in B$ (note that $\widetilde{\nu}$ is a valuation if there is no nonzero element $h$ with $\widetilde{\nu}(h)=\infty$, for instance in case (a)). As in example 2.7, we define $\widetilde{q}_{0}, \ldots, \widetilde{q}_{g} \in B\left[x_{1}, y\right]$ such that $\widetilde{\nu}\left(\widetilde{q}_{r}\right)=\bar{\beta}_{r}$ for $0 \leq r \leq g+1$ as follows: $\widetilde{q}_{0}=x_{1}, \widetilde{q}_{1}=y-\sum_{i<\bar{\beta}_{1}} \lambda_{i}^{\prime}\left(\widetilde{q}_{0}\right)^{\frac{i}{\bar{\beta}_{0}}}$ and, for $1 \leq r \leq g$,

$$
\widetilde{q}_{r+1}=\widetilde{q}_{r}^{n_{r}}-\widetilde{c}_{r} \widetilde{q}_{0}^{b_{r, 0}} \ldots \widetilde{q}_{r-1}^{b_{r, r-1}}-\sum_{\gamma=\left(\gamma_{0}, \ldots, \gamma_{r}\right)} \widetilde{c}_{\gamma} \widetilde{q}_{0}^{\gamma_{0}} \ldots \widetilde{q}_{r}^{\gamma_{r}}, \quad 1 \leq r<g
$$

where $\left\{b_{r, i}\right\}_{i=0}^{r-1}$ are the unique nonnegative integers satisfing $b_{r, i}<n_{i}, 1 \leq i \leq r-1$, and $n_{r} \bar{\beta}_{r}=\sum_{0 \leq i<r} b_{r, i} \bar{\beta}_{i}$, we have $\widetilde{\nu}\left(\widetilde{q}_{0}^{\gamma_{0}} \ldots \widetilde{q}_{r}^{\gamma_{r}}\right)>n_{r} \bar{\beta}_{r}$ for each sequence $\gamma$ of nonnegative integers in the right hand side, and $\widetilde{c}_{r}, \widetilde{c}_{\gamma} \in B, \widetilde{c}_{r} \neq 0$ and $\widetilde{c}_{\gamma} \neq 0$ only for a finite number of $\gamma$ 's. In case (a), we also define $\widetilde{q}_{g+1}$ as in (12); then we have that $\left\{\bar{\beta}_{r}\right\}_{r=0}^{g+1}$ is the minimal generating sequence for the semigroup $\widetilde{\nu}\left(B\left[x_{1}, y\right] \backslash\{0\}\right)$ and $\widetilde{q}_{0}, \ldots, \widetilde{q}_{g+1} \in B\left[x_{1}, y\right]$ is a minimal generating sequence for $\widetilde{\nu}$ ([Sp] theorem 8.6). In case (b), $\widetilde{q}_{g+1} \in B\left[x_{1}, y\right]$, also defined as in (12), defines the kernel of $B\left[x_{1}, y\right] \rightarrow \mathcal{O}_{\widetilde{U}}$.

In case (a), by induction on $r, 1 \leq r \leq g+1$, we will define elements $\left\{q_{r}^{\prime}\right\}_{r=1}^{g+1}$ in $k\left(v_{2}, \ldots, v_{j-1}, x_{1}, y\right)$ more precisely,

$$
q_{r}^{\prime} \in \prod_{r^{\prime}=0}^{r-1} T_{r^{\prime}}^{-1} k\left[v_{2}, \ldots, v_{j-1}, x_{1}, y\right]
$$

where $T_{r^{\prime}}$ is the multiplicative system generated by $q_{r^{\prime}}^{\prime}$, satisfying the following: $q_{0}^{\prime}:=x_{1}$ and, for $1 \leq r \leq g+1$ the image of $q_{r}^{\prime}$ in the fraction field $K\left(\mathcal{O}_{Y, y_{0}}\right)$ of $\mathcal{O}_{Y, y_{0}}$ belongs to $\mathcal{O}_{Y, y_{0}}$ and, if we identify $q_{r}^{\prime}$ with its image, then

$$
\begin{aligned}
& q_{r}^{\prime}=\mu_{r}\left(v_{2}, \ldots, v_{j-1}\right) u^{\bar{\beta}_{r}} \quad \bmod (u)^{\bar{\beta}_{r}+1} \text { for } 1 \leq r \leq g \\
& q_{g+1}^{\prime}=\mu_{g+1}\left(v_{2}, \ldots, v_{j-1}\right) u^{\bar{\beta}_{g+1}} \varrho \quad \bmod (u)^{\bar{\beta}_{g+1}+1}
\end{aligned}
$$

where $\mu_{r}\left(v_{2}, \ldots, v_{j-1}\right)$ is a unit in $R_{j-1}$. In fact, once defined $q_{0}^{\prime}, \ldots, q_{r}^{\prime}$, the element $q_{r+1}^{\prime}$ is defined as follows: let

$$
h_{r, 1}:=q_{0}^{\prime b_{r, 0}} \cdots q_{r-1}^{\prime}{ }^{b_{r, r-1}} P_{r, 1}\left(\frac{\bar{\mu}_{r, 1}\left(q_{r}^{\prime}\right)^{n_{r}}}{q_{0}^{\prime b_{r, 0}} \cdots q_{r-1}^{\prime} b_{r, r-1}}, v_{2}, \ldots, v_{j-1}\right)
$$

where the integers $\left\{b_{r, r^{\prime}}\right\}_{r^{\prime}=0}^{r-1}$ are as in (12), $\bar{\mu}_{r, 1}:=\mu_{1}^{b_{r, 1}} \cdots \mu_{r-1}^{b_{r, r-1}}$ is a unit, and $P_{r, 1} \in k\left[z, v_{2}, \ldots, v_{j-1}\right]$ is such that

$$
P_{r, 1}\left(\mu_{r}^{n_{r}}, v_{2}, \ldots, v_{j-1}\right)=0, \quad \frac{\partial P_{r, 1}}{\partial z}\left(\mu_{r}^{n_{r}}, v_{2}, \ldots, v_{j-1}\right) \text { is a unit in } R_{j-1} .
$$

Then, we have $n_{r} \bar{\beta}_{r}<\nu\left(h_{1}\right) \leq \bar{\beta}_{r+1}$. If $\nu\left(h_{1}\right)=\bar{\beta}_{r+1}$, we set $q_{r+1}^{\prime}:=h_{1}$. If not, we define recursively

$$
h_{r, s}:=q_{0}^{\prime b_{0}^{s}} \cdots q_{r-1}^{\prime b_{r-1}^{s}} P_{r, s}\left(\frac{\bar{\mu}_{r, s} h_{r, s-1}}{q_{0}^{b_{0}^{s}} \cdots q_{r-1}^{b_{r-1}^{s}}}, v_{2}, \ldots, v_{j-1}\right)
$$

where $\left\{b_{r^{\prime}}^{s}\right\}_{r^{\prime}=0}^{r-1}$ are the unique nonnegative integers satisfying $b_{r^{\prime}}^{s}<n_{r^{\prime}}, 1 \leq r^{\prime} \leq$ $r-1$, and $\nu\left(h_{r, s-1}\right)=\sum_{0 \leq r^{\prime} \leq r-1} b_{j, r^{\prime}}^{s} \bar{\beta}_{j, r^{\prime}}, \bar{\mu}_{r, s}:=\mu_{1}^{b_{1}^{s}} \cdots \mu_{r-1}^{b_{r-1}^{s}}$ is a unit, and 
$P_{r, s} \in k\left[z, v_{2}, \ldots, v_{j-1}\right]$ is such that

(15) $P_{r, s}\left(\lambda_{s-1}, v_{2}, \ldots, v_{j-1}\right)=0, \quad \frac{\partial P_{r, s}}{\partial z}\left(\lambda_{s-1}, v_{2}, \ldots, v_{j-1}\right)$ is a unit in $R_{j-1}$

being $\lambda_{s-1} \in R_{j-1}$ the initial form of $h_{r, s-1}$. We have $\nu\left(h_{r, s-1}\right)<\nu\left(h_{r, s}\right) \leq \bar{\beta}_{r+1}$ hence, after a finite number of steps we obtain $s$ such that $\nu\left(h_{r, s}\right)=\bar{\beta}_{r+1}$ and we set $q_{r+1}:=h_{r, s}$ (for more details see [Re4], lemma 3.1).

The elements $q_{r}^{\prime}$ and $\widetilde{q}_{r}$ are related. In fact, for $0 \leq r \leq g+1, q_{r}^{\prime}$ and $\widetilde{q}_{r}$ define the same initial form in an étale covering of a localization of the graded algebra $\operatorname{gr}_{\nu} k\left[v_{2}, \ldots v_{j-1}, x_{1}, y\right]_{\left(x_{1}, y\right)}$. More precisely, there exist $\widetilde{\ell}, \widetilde{h} \in \prod_{0 \leq r^{\prime}<r} T_{r^{\prime}}^{-1} B\left[x_{1}, y\right]$, $\widetilde{\ell}$ being a unit and $\widetilde{\nu}(\widetilde{h})>\bar{\beta}_{r}$ such that $q_{r}^{\prime}=\widetilde{q}_{r} \cdot \widetilde{\ell}+\widetilde{h}$.

3.4. Recall the expression in (11). Fixed $j, 2 \leq j \leq \delta$, we apply the previous study to

$$
\begin{aligned}
& x_{1} \mapsto u_{1}^{m_{1}} \\
& x_{j} \mapsto \sum_{1 \leq i \leq m_{j}} \lambda_{j, i}\left(u_{2}, \ldots, u_{j-1}\right) u_{1}^{i}+u_{1}^{m_{j}} u_{j} .
\end{aligned}
$$

Let $B_{j-1}$ be a domain which is an étale extension of $k\left[u_{2}, \ldots, u_{j-1}\right]$ and contains $\lambda_{j, i}\left(u_{2}, \ldots, u_{j-1}\right), m_{1} \leq i \leq m_{j}$, let $\widetilde{\nu}_{j}$ be the valuation on $B_{j-1}\left[x_{1}, x_{j}\right]$ extending $\nu$ and let $\left\{\bar{\beta}_{j, r}\right\}_{r=0}^{g_{j}+1}$ the minimal generating sequence for the semigroup $\widetilde{\nu}_{j}\left(B_{j-1}\left[x_{1}, x_{j}\right] \backslash\{0\}\right)$. Let $\left\{\widetilde{q}_{j, r}\right\}_{r=0}^{g_{j}+1} \in B_{j-1}\left[x_{1}, x_{j}\right]$ be a minimal generating sequence for $\widetilde{\nu}_{j}$, and $\left\{q_{j, r}^{\prime}\right\}_{r=0}^{g_{j}+1} \in k\left(u_{2}, \ldots, u_{j-1}, x_{1}, x_{j}\right)$ defined as in 3.3 .

Consider the following sets with the lexicographic order

$\mathcal{J}^{*}:=\{(1,0)\} \cup\left\{(j, r) / 2 \leq j \leq \delta, 1 \leq r \leq g_{j}\right\}, \quad \mathcal{J}:=\mathcal{J}^{*} \cup\left\{\left(j, g_{j}+1\right) / 2 \leq j \leq \delta\right\}$.

Applying the argument in 3.3 and arguing by induction on $(j, r) \in \mathcal{J}$, we can define elements $\left\{q_{j, r}\right\}_{(j, r) \in \mathcal{J}}$,

$$
q_{j, r} \in \prod_{\substack{\left(j^{\prime}, r^{\prime}\right) \in \mathcal{J}^{*} \\\left(j^{\prime}, r^{\prime}\right)<(j, r)}} T_{j^{\prime}, r^{\prime}}^{-1} k\left[x_{1}, \ldots, x_{j}\right]
$$

where $T_{j^{\prime}, r^{\prime}}$ is the multiplicative system generated by $q_{j^{\prime}, r^{\prime}}$, satisfying the following: $q_{1,0}:=x_{1}$ and, for $(j, r) \in \mathcal{J}$, the image of $q_{j, r}$ in the fraction field $K\left(\mathcal{O}_{Y, y_{0}}\right)$ of $\mathcal{O}_{Y, y_{0}}$ belongs to $\mathcal{O}_{Y, y_{0}}$ and, if we identify $q_{j, r}$ with its image, then

$$
\begin{array}{ll}
q_{j, r} & =\mu_{j, r}\left(u_{2}, \ldots, u_{j-1}\right) u^{\bar{\beta}_{j, r}} \quad \bmod (u)^{\bar{\beta}_{j, r}+1} \text { for } 1 \leq r \leq g_{j} \\
q_{j, g_{j}+1} & =\mu_{j, g_{j}+1}\left(u_{2}, \ldots, u_{j-1}\right) u^{\bar{\beta}_{j, g_{j}+1}} u_{j} \quad \bmod (u)^{\bar{\beta}_{j, g_{j}+1}+1}
\end{array}
$$

where $\mu_{j, r}\left(u_{2}, \ldots, u_{j-1}\right)$ is a unit in $k<u_{2}, \ldots, u_{j-1}>$. Besides, if $b_{j, 0}, \ldots, b_{j, g_{j}}$ are the unique nonnegative integers satisfying $b_{j, r}<n_{j, r}, 1 \leq r \leq g_{j}$, and $\bar{\beta}_{j, g_{j}+1}=$ $\sum_{0 \leq i \leq g_{j}} b_{j, r} \bar{\beta}_{j, r}$, and we set $q_{j, 0}:=q_{1,0}=x_{1}$, then, identifying $q_{j, r}$ with its image in $\overline{\mathcal{O}}_{Y, y_{0}}$, we have

$$
\frac{q_{j, g_{j}+1}}{q_{j, 0}^{b_{j, 0}} \ldots q_{j, g_{j}}^{b_{j, g_{j}}}}=v_{j} \in \mathcal{O}_{Y, y_{0}} .
$$

where $v_{j}=\gamma_{j} u_{j} \bmod (u)$, being $\gamma_{j}$ a unit in $k<u_{2}, \ldots, u_{j-1}>$. In particular note that $\left.k<u_{2}, \ldots, u_{j}\right\rangle=k<v_{2}, \ldots, v_{j}>$. Note also that $q_{j, r}$ is obtained from $q_{j, r}^{\prime}$ by replacing $v_{j^{\prime}}$ by $\frac{q_{j^{\prime}, g_{j^{\prime}}+1}}{q_{j^{\prime}, 0}^{b_{j^{\prime}, 0} \ldots b_{j^{\prime}, g_{j^{\prime}}}}}$, for $1 \leq j^{\prime}<j$. We will denote $\left\{P_{j, r, s}\right\}_{s}$ the polynomials in $k\left[z, v_{2}, \ldots, v_{j-1}\right]$ defined in order to obtain $q_{j, r+1}^{\prime}$ from $q_{j, r}^{\prime}$, hence satisfying (14) (resp. (15)) for $s=1$ (resp. $s>1$ ). The elements $\left\{q_{j, r}\right\}_{(j, r) \in \mathcal{J}}$ are 
called a system of transverse generators for $\eta: Y \rightarrow \mathbb{A}_{k}^{d}$ with respect to $E$.

3.5. Finally, for every element $q \in \mathcal{O}_{Y, y_{0}}$ which is the image of an element in the fraction field of $k\left[x_{1}, \ldots, x_{d}\right]$, i.e. we can identify $q=l / g$ where $l, g \in k\left[x_{1}, \ldots, x_{d}\right]$, we can define $\left\{\bar{Q}_{n}\right\}_{n \geq 0}$ in $\mathcal{O}_{\left(\mathbb{A}^{d}\right)_{\infty}, P_{e E}^{\mathbb{A}^{d}}}$ such that, in the ring $\mathcal{O}_{Y_{\infty}, P_{e E}^{Y}}$, we have

$$
Q_{n} \equiv \bar{Q}_{n} \bmod P_{e E}^{Y}
$$

More precisely, since $P_{e E}^{Y}$ is a stable point and the image of $g$ in $\mathcal{O}_{Y, y_{0}}$ is nonzero, there exists $c \in \mathbb{N}$ such that $G_{0}, \ldots, G_{c-1} \in P_{e E}^{Y}, G_{c} \notin P_{e E}^{Y}$. Hence we have

$$
G_{c} Q_{n}+\ldots+G_{n+c} Q_{e} \equiv L_{n+c} \bmod P_{e E}^{Y} \text { for } n \geq 0
$$

((14) in [Re3] proof of prop. 4.1) and we can define by recurrence $\bar{Q}_{n} \in S^{-1} \mathcal{O}_{\mathbb{A}_{\infty}^{d}}$, where $S$ is the multiplicative system generated by $G_{c}$, satisfying (19) (see also lemma 4.1 in $[\operatorname{Re} 4])$.

Applying this to each $q_{j, r}$, we obtain $\bar{Q}_{j, r ; n} \in \mathcal{O}_{\left(\mathbb{A}^{d}\right)_{\infty}, P_{e E}^{\mathbb{A}^{d}}}, n \geq 0$, such that $Q_{j, r ; n} \equiv \bar{Q}_{j, r ; n}$ modulo $P_{e E}^{Y}$. More precisely,

$$
\bar{Q}_{j, r ; n} \in \prod_{\substack{\left(j^{\prime}, r^{\prime}\right) \in \mathcal{J}^{*} \\\left(j^{\prime}, r^{\prime}\right)<(j, r)}} \bar{T}_{j^{\prime}, r^{\prime}}^{-1} \quad k\left[x_{1}, \ldots, x_{j}\right]_{\infty}
$$

where $k\left[x_{1}, \ldots, x_{j}\right]_{\infty}$ denotes $\mathcal{O}_{\left(\operatorname{Spec} k\left[x_{1}, \ldots, x_{j}\right]\right)_{\infty}}$ and $\bar{T}_{j^{\prime}, r^{\prime}}$ is the multiplicative system generated by $\bar{Q}_{j^{\prime}, r^{\prime} ; e \bar{\beta}_{j^{\prime}, r^{\prime}}}$. Then, let

$$
\mathcal{Q}:=\left\{\bar{Q}_{j, r ; n}\right\}_{(j, r) \in \mathcal{J}, e n_{j, r-1} \bar{\beta}_{j, r-1} \leq n \leq e \bar{\beta}_{j, r}-1} .
$$

It is clear (see (17)) that $(\mathcal{Q}) \mathcal{O}_{\left(\mathbb{A}^{d}\right)_{\infty}, P_{e E}^{\mathbb{A}^{d}}} \subseteq P_{e E}^{\mathbb{A}^{d}} \mathcal{O}_{\left(\mathbb{A}^{d}\right)_{\infty}, P_{e E}^{\mathbb{A}^{d}}}$. Besides, note that, applying (6), (11) and, for the last equality, also (9), we have

$$
\begin{aligned}
\sharp \mathcal{Q} & =\sum_{j=2}^{\delta}\left(e \bar{\beta}_{j, 1}+e\left(\bar{\beta}_{j, 2}-n_{j, 1} \bar{\beta}_{j, 1}\right)+\ldots+e\left(\bar{\beta}_{j, g_{j}+1}-n_{j, g_{j}} \bar{\beta}_{j, g_{j}}\right)\right)= \\
& =e \sum_{j=2}^{\delta}\left(\beta_{j, 1}+\left(\beta_{j, 2}-\beta_{j, 1}\right)+\ldots+\left(\beta_{j, g_{j}+1}-\beta_{j, g_{j}}\right)\right)= \\
& =e \sum_{j=2}^{\delta} \beta_{j, g_{j}+1}=e \sum_{j=2}^{\delta} m_{j}=e\left(k_{E}\left(\mathbb{A}_{k}^{d}\right)+1\right)=e\left(\widehat{k}_{E}(X)+1\right) .
\end{aligned}
$$

and recall that $\mathcal{O}_{\left(\mathbb{A}^{d}\right)_{\infty}, P_{e E}^{\AA^{d}}}$ is a regular local ring of dimension $e\left(k_{E}\left(\mathbb{A}_{k}^{d}\right)+1\right)$ (see (xiii)). In [Re4] we have proved that $\mathcal{Q}$ is a regular system of parameters of $\mathcal{O}_{\left(\mathbb{A}^{d}\right)_{\infty}, P_{e E}^{\mathbb{A}^{d}}}$; then $\mathcal{Q}$ is called regular system of parameters of $\mathcal{O}_{\left(\mathbb{A}^{d}\right)_{\infty}, P_{E}^{\mathbb{A}^{d}}}$ as-

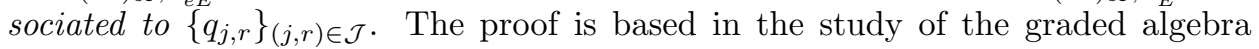
$g r_{\nu_{E}} k\left[x_{1}, \ldots, x_{d}\right]$. In fact, the main idea in the proof is to show that $(\mathcal{Q}) \mathcal{O}_{\left(\mathbb{A}^{d}\right)_{\infty}, P_{e E}^{\mathbb{A}^{d}}}$ is a prime ideal and it follows from the following: It is proved that, modulo étale extension, $\mathcal{O}_{\left(\mathbb{A}^{d}\right)_{\infty}, P_{e E}^{\mathbb{A}^{d}}} /(\mathcal{Q})$ is isomorphic to a polynomial ring in countable many variables over certain localization of $g r_{\nu_{E}} k\left[x_{1}, \ldots, x_{d}\right]$. Since $g r_{\nu_{E}} k\left[x_{1}, \ldots, x_{d}\right]$ is a domain because $\nu_{E}$ is a valuation it follows that $\mathcal{O}_{\left(\mathbb{A}^{d}\right)_{\infty}, P_{e E}^{\AA^{d}}} /(\mathcal{Q})$ is a domain (see [Re4], theorem 4.8).

More generally, let $\widetilde{q}_{0}, \ldots, \widetilde{q}_{g+1} \in B\left[x_{1}, y\right]$ be as in 3.3 , and let us define $\widetilde{\mathcal{Q}}:=$ $\left\{\widetilde{Q}_{r ; n}\right\}_{0 \leq r \leq g+1, e n_{j, r-1} \bar{\beta}_{j, r-1} \leq n \leq e \bar{\beta}_{j, r}-1}$ where $\widetilde{Q}_{r ; n} \in B\left[x_{1}, y\right]_{\infty}$ and $\widetilde{L}:=\prod_{r=0}^{g} \widetilde{Q}_{r ; e \bar{\beta}_{r}}$. Then $(\widetilde{\mathcal{Q}})$ is a prime ideal of $\left(B\left[x_{1}, y\right]_{\infty}\right) \widetilde{L}([\operatorname{Re} 4]$, prop. 4.5$)$.

In order to study the ring $\widehat{\mathcal{O}_{X_{\infty}, P_{e E}}}$ we may first suppose that the irreducible component $X_{0}$ of $X$ where the valuation $\nu$ is defined is analytically irreducible. In fact, there exists an étale morphism $\widetilde{X} \rightarrow X$ such that each irreducible component of $\widetilde{X}$ is analytically irreducible. Hence, there exists an irreducible component $\widetilde{X}_{0}$ of $\widetilde{X}$ whose image is $X_{0}$, and there exists a divisorial valuation $\widetilde{\nu}$ on $\widetilde{X}_{0}$ extending 
$\nu$. Let $\widetilde{P}_{e}$ be the stable point on $\widetilde{X}_{0}$ defined by $\widetilde{\nu}$ and $e$, whose image is $P_{e E}$. Then $\widehat{\mathcal{O}_{\widetilde{X}_{0}, \widetilde{P}_{e}}} \cong \widehat{\mathcal{O}_{X_{0}, P_{e E}}} \otimes_{\kappa\left(P_{e E}\right)} \kappa\left(\widetilde{P}_{e}\right)$ (see (xii)). So, assume that $X_{0}$ is analytically irreducible. We will embed $X_{0}$ in a complete intersection scheme $X^{\prime} \subseteq \mathbb{A}_{k}^{M}$ of dimension $d=\operatorname{dim} X_{0}$. For any such $X^{\prime}$ we have

$$
\mathcal{O}_{\left(X_{\infty}\right)_{\mathrm{red}}, P_{e E}} \cong \mathcal{O}_{\left(X_{\infty}^{\prime}\right)_{\mathrm{red}}, P_{e E}} \quad \text { and } \quad \widehat{\mathcal{O}_{\left(X_{\infty}\right), P_{e E}}} \cong \widehat{\mathcal{O}_{\left(X_{\infty}^{\prime}\right), P_{e E}}}
$$

where we also denote by $P_{e E}$ the point induced by $P_{e E}^{X}$ in $X_{\infty}^{\prime}$ or in $\left(X_{\infty}^{\prime}\right)_{\text {red }}$ (see (ii) and (ix) in 2.3).

Proposition 3.6. Assume that char $k=0$. Let $X_{0}$ be a reduced separated $k$ scheme of finite type. Assume that $X_{0}$ is analytically irreducible. Let $\nu=\nu_{E}$ be a divisorial valuation on $X_{0}$ and let $e \in \mathbb{N}$. Then, there exist a complete intersection scheme

$$
X^{\prime}=\operatorname{Spec} k\left[y_{1}, \ldots, y_{N}\right] /\left(f_{d+1}, \ldots, f_{N}\right) \subseteq \mathbb{A}_{k}^{N}
$$

which contains $X_{0}$, and of dimension $d=\operatorname{dim} X_{0}$, and elements $\left\{z_{l, s}\right\}_{d+1 \leq l \leq N, 1 \leq s \leq g_{l}}$ in $k\left[y_{1}, \ldots, y_{N}\right]$, such that, if given $g \in k\left[y_{1}, \ldots, y_{N}\right]$ we denote $\nu(g)$ the $\nu$-value of the class of $g$ in $\mathcal{O}_{X_{0}}$, then the following holds:

(a) For $d+1 \leq l \leq N, 1 \leq s \leq g_{l}$ let $\bar{\alpha}_{l, s}:=\nu\left(z_{l, s}\right)$ and let

$$
\mathcal{Z}=\cup_{l=d+1}^{N} \mathcal{Z}_{l} \quad \text { where } \quad \mathcal{Z}_{l}:=\left\{Z_{l, s ; n}\right\}_{\substack{1 \leq s \leq g_{l} \\ 0 \leq n<e \bar{\alpha}_{l, s}}}
$$

being $Z_{j, r ; n} \in k\left[y_{1}, \ldots, y_{N}\right]_{\infty}$. Then there exists $G \in \mathcal{O}_{\left(\mathbb{A}^{N}\right)_{\infty}}$ such that $(\mathcal{Q} \cup \mathcal{Z})\left(\mathcal{O}_{\left(\mathbb{A}^{N}\right)_{\infty}}\right)_{G}$ is a prime ideal and

$$
P_{e E}^{X^{\prime}} \mathcal{O}_{X_{\infty}^{\prime}, P_{e E}^{X^{\prime}}}=(\mathcal{Q} \cup \mathcal{Z}) \mathcal{O}_{X_{\infty}^{\prime}, P_{e E}^{X^{\prime}}}
$$

(b) For $d+1 \leq l \leq N, f_{l}=f_{l}\left(y_{1}, \ldots, y_{d}, y_{l}\right) \in k\left[y_{1}, \ldots, y_{d}, y_{l}\right]$ satisfies:

(i) $\nu\left(\operatorname{Jac}\left(f_{l}\right)\right)=\nu\left(\frac{\partial f_{l}}{\partial y_{l}}\right)$; set $\epsilon_{l}:=\nu\left(\operatorname{Jac}\left(f_{l}\right)\right)$,

(ii) for all $n \geq 0$, the class of $\frac{\partial F_{l ; e \epsilon_{l}+n}}{\partial Y_{l ; n}}$ in $\mathcal{O}_{X_{\infty}^{\prime}, P_{e E}}$ is a unit and, for $n^{\prime}>n$, the class of $\frac{\partial F_{l ; e \epsilon_{l}+n}}{\partial Y_{l ; n^{\prime}}}$ in $\mathcal{O}_{X_{\infty}^{\prime}, P_{e E}}$ belongs to $P_{e E} \mathcal{O}_{X_{\infty}^{\prime}, P_{e E}}$. Besides, if we denote $f_{l, l}^{\prime}:=\frac{\partial f_{l}}{\partial y_{l}}$ then the class of $\frac{\partial F_{l ; e \epsilon_{l}+n}}{\partial Y_{l ; n}}-F_{l, l ; e \epsilon_{l}}^{\prime}$ in $\mathcal{O}_{X_{\infty}^{\prime}, P_{e E}}$ belongs to $P_{e E}$.

(iii) there exists $L \in \mathcal{O}_{\mathbb{A}_{\infty}^{d}}=k\left[x_{1}, \ldots, x_{d}\right]_{\infty}, L \notin P_{e E}^{\mathbb{A}^{d}}$, such that the elements $F_{l ; 0}, \ldots, F_{l ; e \epsilon_{l}-1}$ belong to $\left(\mathcal{Q} \cup \mathcal{Z}_{l}\right)^{2}\left(\mathcal{O}_{\left(\mathbb{A}_{k}^{N}\right)_{\infty}}\right)_{L}$.

Proof. Let $\pi: Y \rightarrow X_{0}, \rho: X_{0} \rightarrow \mathbb{A}_{k}^{d}$ and $\eta=\rho \circ \pi: Y \rightarrow \mathbb{A}_{k}^{d}$ be as in the beginning of this section. Let us consider an étale morphism $\widetilde{U} \rightarrow U$ as in 3.2 and keep the notation in 3.2. From the discussion in 3.2, 3.3 and 3.4 it follows that there exist $\left\{u, v_{2}, \ldots, v_{d}\right\} \in \mathcal{O}_{\widetilde{U}}$ and $\left\{x_{1}, \ldots, x_{d}, x_{d+1}, \ldots, x_{N}\right\} \in \mathcal{O}_{X}$ such that, after replacing $v_{i}$ by $v_{i}+c_{i}$ where $c_{i} \in k, 2 \leq i \leq d$, the following property holds for the points $y_{0}$ in an open subset of $\widetilde{E}:\left\{u, v_{2}, \ldots, v_{d}\right\}$ (resp. $\left\{x_{1}, \ldots, x_{d}\right\}$ ) is a regular system of parameters of $\mathcal{O}_{\widetilde{U}, y_{0}}$ (resp. $\left.\mathcal{O}_{\mathbb{A}_{k}^{d}, \eta\left(y_{0}\right)}\right)$ and $\left\{x_{1}, \ldots, x_{d}, x_{d+1}, \ldots, x_{N}\right\}$ generate the maximal ideal of $\mathcal{O}_{X_{0}, \pi\left(y_{0}\right)}$ such that:

(i) The local expression for $\eta$ in (11) holds for the regular system of parameters $\left\{u, v_{2}, \ldots, v_{d}\right\}$ of $\mathcal{O}_{\widetilde{U}, y_{0}}$ and $\left\{x_{1}, \ldots, x_{d}\right\}$ of $\mathcal{O}_{\mathbb{A}^{d}, \eta\left(y_{0}\right)}$ (i.e. in (11) replace $u_{1}$ by $u, u_{i}$ by $v_{i}$ for $2 \leq i \leq \delta$, and set $v_{i}=u_{i}$ for $\left.\delta<i \leq d\right)$.

(ii) There exists a system of transverse generators $\left\{q_{j, r}\right\}_{(j, r) \in \mathcal{J}}$ for $\eta: Y \rightarrow \mathbb{A}_{k}^{d}$ with respect to $E$, hence satisfying (16), (17) and (18). 
(iii) For $d+1 \leq l \leq N$, the image of $x_{l}$ in $\mathcal{O}_{Y, y_{0}}$ is expressed as

$$
x_{l}=\sum_{m_{1} \leq i} \lambda_{l, i}(\underline{v}) u^{i}
$$

where, $\underline{v}:=\left(v_{2}, \ldots, v_{d}\right)$ and

$$
\begin{aligned}
& \lambda_{l, i}(\underline{v}) \quad \in k<\underline{v}>\cap \mathcal{O}_{\widetilde{U}, y_{0}} \\
& \lambda_{l, i}(\underline{v}) \quad \in k<v_{2}, \ldots, v_{j-1}>\cap \mathcal{O}_{\widetilde{U}, y_{0}} \quad \text { if } i<m_{j} \quad \text { for } 2 \leq j \leq \delta
\end{aligned}
$$

(recall (9) for the second assertion in (22)).

Fix $l, d+1 \leq l \leq N$. Let $\bar{\beta}_{l, 0}, \ldots, \bar{\beta}_{l, g_{l}}$ be a minimal system of generators of the semigroup defined by the restriction $\nu_{l}$ of $\nu_{E}$ to $k(\underline{v})\left[x_{1}, x_{l}\right]_{\left(x_{1}, x_{l}\right)}$. Let $e_{l, r}=$ g.c.d. $\left\{\bar{\beta}_{l, 0}, \ldots, \bar{\beta}_{l, r}\right\}, 0 \leq r \leq g_{l}, n_{l, r}=\frac{e_{l, r-1}}{e_{l, r}}, 1 \leq r \leq g_{l}$, and let $\beta_{l, 0}, \ldots, \beta_{l, g_{l}}$ be defined by $\bar{\beta}_{l, r}-n_{l, r-1} \bar{\beta}_{l, r-1}=\beta_{l, r}-\beta_{l, r-1}$ as in (6). Consider $h \in k[\underline{v}]$ such that $k[\underline{v}]_{h}$ is contained in the $\operatorname{ring} \mathcal{O}_{\widetilde{U}}$ and consider the morphism $\theta_{l}: \widetilde{U} \rightarrow$ Spec $k[\underline{v}]_{h}\left[x_{1}, y\right]$ given by

$$
\begin{aligned}
x_{1} & \mapsto u^{m_{1}} \\
y & \mapsto \sum_{m_{1} \leq i} \lambda_{l, i}(\underline{v}) u^{i}
\end{aligned}
$$

Since $X_{0}$ is analytically irreducible, there exists a domain $B_{l}$ such that $B_{l}\left[x_{1}, y\right]$ is an étale extension of $k[\underline{v}]_{h}\left[x_{1}, y\right]$ and there exist $x_{1}^{\prime}, y^{\prime} \in B_{l}\left[x_{1}, y\right]$ with

$$
x_{1}^{\prime}=\gamma_{1} x_{1}, \quad y^{\prime}=\gamma_{l} y \quad \text { where } \gamma_{1}, \gamma_{l} \in B_{l}\left[x_{1}, y\right] \quad \text { are units }
$$

and $u^{\prime}=\mu u$ where $\mu$ is a unit in an étale extension of $k[\underline{v}]_{h}[u]$, such that the induced morphism $\widetilde{\theta}_{l}: \widetilde{\widetilde{U}} \rightarrow \operatorname{Spec} B_{l}\left[x_{1}^{\prime}, y^{\prime}\right]$, being $\widetilde{\widetilde{U}} \rightarrow \widetilde{U}$ étale, is given by

$$
\begin{aligned}
x_{1}^{\prime} & \mapsto\left(u^{\prime}\right)^{m_{1}} \\
y^{\prime} & \mapsto \sum_{m_{1} \leq i \leq m} \lambda_{l, i}^{\prime}\left(u^{\prime}\right)^{i}
\end{aligned}
$$

where $\lambda_{l, i}^{\prime} \in B_{l}$ for $m_{1} \leq i \leq m$ (see remark 2.8). Let $\widetilde{q}_{l, 0}, \ldots, \widetilde{q}_{l, g_{l}}, \widetilde{q}_{l, g_{l}+1} \in$ $B_{l}\left[x_{1}^{\prime}, y^{\prime}\right]$ be the elements defined as in 3.3 applied to the previous expression, hence case (b) in 3.3. Hence $\widetilde{q}_{l, g_{l}+1}$ defines the kernel of $B_{l}\left[x_{1}, y\right] \rightarrow \mathcal{O}_{\widetilde{U}}$, i.e.

$$
B_{l}\left[x_{1}, x_{l}\right] \cong B_{l}\left[x_{1}, y\right] /\left(\widetilde{q}_{l, g_{l}+1}\right) .
$$

Thus $\widetilde{q}_{l, g_{l}+1}$ defines an equation of a plane curve in Spec $L_{l}\left[x_{1}^{\prime}, y^{\prime}\right]$, where $L_{l}$ is a field extension of $k$ containing $\lambda_{l, i}^{\prime}$ for $m_{1} \leq i \leq m$, which is analytically irreducible, and $\widetilde{q}_{l, 1}, \ldots, \widetilde{q}_{l, g_{l}}$ are its approximate roots. Let us also consider the following elements in $k[\underline{v}]_{h}\left[x_{1}, y\right]$ : Let $f_{0}^{\prime}:=\widetilde{q}_{0}=x_{1}$ and, for $1 \leq r \leq g+1$, let us define $f_{r}^{\prime}$ to be an irreducible polynomial in $k[\underline{v}]_{h}\left[x_{1}, y\right]$ defining the contracted ideal of $\left(\widetilde{q}_{r}\right) B_{l}\left[x_{1}, y\right]$ to $k[\underline{v}]_{h}\left[x_{1}, y\right]$. Set $f_{l}^{\prime}:=f_{l, g_{l}+1}$ and note that we have

$$
f_{l}^{\prime}\left(\underline{v}, x_{1}, y\right)=\widetilde{q}_{l, g_{l}+1} \cdot \widetilde{h}
$$

where $\widetilde{h} \in B_{l}\left[x_{1}, y\right]$ and $\widetilde{q}_{l, g_{l}+1}$ does not divide $\widetilde{h}$. Let

$$
C_{l}:=\operatorname{Spec} k[\underline{v}]_{h}\left[x_{1}, y\right] /\left(f_{l}^{\prime}\right) \quad \widetilde{C}_{l}:=\operatorname{Spec} B_{l}\left[x_{1}, y\right] /\left(\widetilde{q}_{l, g_{l}+1}\right) .
$$

Note that the induced morphism $\widetilde{C}_{l} \rightarrow C_{l}$ is étale.

We consider now the spaces of arcs of $C_{l}, \widetilde{C}_{l}$. Let $\widetilde{\nu}$ be a divisorial valuation on $B_{l}\left[x_{1}, y\right] /\left(\widetilde{q}_{l, g_{l}+1}\right)$ extending $\nu_{l}$, hence $\widetilde{\nu}(\lambda)=0$ for all $\lambda \in B_{l}$ (recall that $\nu_{l}\left(v_{j}\right)=0,2 \leq j \leq d$ ) and let $P_{l}^{\prime}$ (resp. $\widetilde{P}_{l}$ ) be the stable point of $\mathcal{O}_{\left(C_{l}\right)_{\infty}}$ (resp.

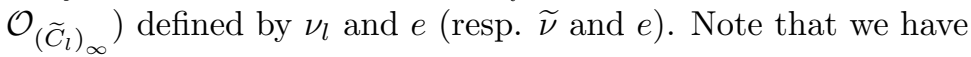

$$
\widehat{\mathcal{O}}_{\left(C_{l}\right)_{\infty}, P_{l}^{\prime}} \prec \widehat{\mathcal{O}}_{\left(\widetilde{C}_{l}\right)_{\infty}, \widetilde{P}_{l}}
$$


i.e. the ring on the right hand side dominates the ring on the left hand side. Following 3.5, let $\widetilde{\mathcal{Q}}_{l}:=\left\{\widetilde{Q}_{l, r ; n}\right\}_{0 \leq r \leq g_{l}, e n_{j, r-1} \bar{\beta}_{j, r-1} \leq n \leq e \bar{\beta}_{l, r}-1}$, then $\left(\widetilde{\mathcal{Q}}_{l}\right)$ defines a prime ideal $\widetilde{\mathbb{P}}_{l}$ in $\left(B_{l}\left[x_{1}, y\right]_{\infty}\right)_{\widetilde{L}}$, where $\widetilde{L}=\prod_{r=0}^{g} \widetilde{Q}_{r ; e \bar{\beta}_{r}}$, and we have

$$
\left(\widetilde{\mathcal{Q}}_{l}\right) \mathcal{O}_{\left(\widetilde{C}_{l}\right)_{\infty}, \widetilde{P}_{l}}=\widetilde{P}_{l} \mathcal{O}_{\left(\widetilde{C}_{l}\right)_{\infty}, \widetilde{P}_{l}}
$$

(this argument has already been applied in Example 2.7, it is based on [Re4], prop. 4.5 , see also 3.5 ). Besides $\widetilde{\mathbb{P}}_{l}$ is a stable point of $B_{l}\left[x_{1}, y\right]_{\infty}$, since $\widetilde{\mathcal{Q}}_{l}$ is a finite set. Let $\mathbb{P}_{l}^{\prime}$ be the image of $\widetilde{\mathbb{P}}_{l}$ in $\left(\operatorname{Spec} k[\underline{v}]_{h}\left[x_{1}, y\right]\right)_{\infty}$. Since the morphism $k[\underline{v}]_{h}\left[x_{1}, y\right]_{\left(x_{1}, y\right)} \rightarrow B_{l}\left[x_{1}, y\right]_{\left(x_{1}, y\right)}$ is étale, $\mathbb{P}_{l}^{\prime}$ is a stable point and we have

$$
\left(B_{l}\left[\widehat{\left.x_{1}, y\right]_{\infty}}\right)_{\widetilde{\mathbb{P}}_{l}} \cong\left(k[\underline{v}]_{h} \widehat{\left[x_{1}, y\right.}\right]_{\infty}\right)_{\mathbb{P}_{l}^{\prime}} \otimes_{\kappa\left(\mathbb{P}_{l}^{\prime}\right)} \kappa\left(\widetilde{\mathbb{P}}_{l}\right)
$$

([Re4] prop. 2.5, see (xii)). Let $\mathcal{F}_{l}^{\prime}:=\left\{F_{l, r ; n}^{\prime}\right\}_{0 \leq r \leq g_{l}, 0 \leq n<\nu\left(f_{l, r}^{\prime}\right)}$ and let $L^{\prime}=$ $H_{0} \cdot \prod_{r=0}^{g} F_{r ; e \nu\left(f_{r}^{\prime}\right)}^{\prime}$, then $\left(\mathcal{F}_{l}^{\prime}\right)\left(k\left[\underline{v}, x_{1}, y\right]_{\infty}\right)_{L^{\prime}}$ is a prime ideal ([Re4], proof of prop. 4.5 , see 3.5$)$ and we have

$$
\left(\mathcal{F}_{l}^{\prime}\right)\left(k[\underline{v}]_{h}\left[x_{1}, y\right]_{\infty}\right)_{L^{\prime}}=\mathbb{P}_{l}^{\prime}\left(k[\underline{v}]_{h}\left[x_{1}, y\right]_{\infty}\right)_{L^{\prime}}
$$

and

$$
\left(\mathcal{F}_{l}^{\prime}\right) \mathcal{O}_{\left(C_{l}\right)_{\infty}, P_{l}^{\prime}}=P_{l}^{\prime} \mathcal{O}_{\left(C_{l}\right)_{\infty}, P_{l}^{\prime}}
$$

Now, for $\widetilde{q}_{l, g_{l}+1}$, we have

(a.1) $\widetilde{\nu}\left(J a c\left(\widetilde{q}_{l, g_{l}+1}\right)\right)=\widetilde{\nu}\left(\frac{\partial \widetilde{q}_{l, g_{l}+1}}{\partial y}\right)=\widetilde{\nu}\left(\frac{\partial \widetilde{q}_{l, g_{l}+1}}{\partial y^{\prime}}\right)=\left(n_{l, g_{l}}-1\right) \bar{\beta}_{l, g_{l}}+\ldots+$ $+\left(n_{1}-1\right) \bar{\beta}_{l, 1}=n_{l, g_{l}} \bar{\beta}_{l, g_{l}}-\beta_{l, g_{l}}$. Set $\widetilde{\epsilon}:=n_{l, g_{l}} \bar{\beta}_{l, g_{l}}-\beta_{l, g_{l}}$, then:

(b.1) for all $n \geq 0$, the class of $\frac{\partial \widetilde{Q}_{l, g_{l}+1 ; e \tilde{\epsilon}+n}}{\partial Y_{n}^{\prime}}$ in $\mathcal{O}_{\left(\widetilde{C}_{l}\right)_{\infty}, \widetilde{P}_{l}}$ is equal to the class of

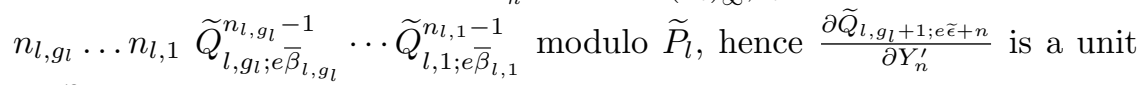
in $\mathcal{O}_{\left(\widetilde{C}_{l}\right)_{\infty}, \widetilde{P}_{l}}$,

(c.1) for $n^{\prime}>n$, the class of $\frac{\partial \widetilde{Q}_{l, g_{l}+1 ; e \tilde{\epsilon}+n}}{\partial Y_{n^{\prime}}^{\prime}}$ in $\mathcal{O}_{\left(\widetilde{C}_{l}\right)_{\infty}, \widetilde{P}_{l}}$ belongs to $\widetilde{P}_{l} \mathcal{O}_{\left(\widetilde{C}_{l}\right)_{\infty}, \widetilde{P}_{l}}$; . Therefore:

(b'.1) for all $n \geq 0$, the class of $\frac{\partial \widetilde{Q}_{l, g_{l}+1 ; e \tilde{\epsilon}+n}}{\partial Y_{n}}$ in $\mathcal{O}_{\left(\widetilde{C}_{l}\right)_{\infty}, \widetilde{P}_{l}}$ is is a unit in $\mathcal{O}_{\left(\widetilde{C}_{l}\right)_{\infty}, \widetilde{P}_{l}}$

(c'.1) for $n^{\prime}>n$, the class of $\frac{\partial \widetilde{Q}_{l, g_{l}+1 ; e \tilde{\epsilon}+n}}{\partial Y_{n^{\prime}}}$ in $\mathcal{O}_{\left(\widetilde{C}_{l}\right)_{\infty}, \widetilde{P}_{l}}$ belongs to $\widetilde{P}_{l} \mathcal{O}_{\left(\widetilde{C}_{l}\right)_{\infty}, \widetilde{P}_{l}}$;

(d.1) $\widetilde{Q}_{l, g_{l}+1 ; 0}, \ldots, \widetilde{Q}_{l, g_{l}+1 ; e \widetilde{\epsilon}-1}$ belong to $\left(\widetilde{\mathcal{Q}}_{l}\right)^{2} B_{l}\left[\left[x_{1}, y\right]\right]_{\infty}$.

In fact, to prove (d.1) we argue by recurrence, and prove that, for $1 \leq r \leq g_{l}+1$,

$$
\widetilde{Q}_{l, r ; n} \in\left(\left\{\widetilde{Q}_{l, r^{\prime} ; n}\right\}_{0 \leq r^{\prime} \leq r-1,0 \leq n \leq \bar{\beta}_{l, r^{\prime}}-1}\right)^{2} B_{l}\left[x_{1}, y\right]_{\infty}
$$

for $0 \leq n<e\left(\left(n_{l, r-1}-1\right) \bar{\beta}_{l, r-1}+\ldots+\left(n_{l, 1}-1\right) \bar{\beta}_{l, 1}\right)=e\left(\bar{\beta}_{l, r}-\beta_{l, r}\right)$. Now, from $(24)$ and $(25)$ we obtain that $F_{l ; 0}^{\prime}, \ldots, F_{l ; e \epsilon^{\prime}-1}^{\prime}$ belong to $\left(\mathcal{F}^{\prime}{ }_{l}\right)^{2}\left(k\left[\underline{v}, x_{1}, y\right]_{\infty}\right)_{\mathbb{P}_{l}^{\prime}}$, where $\epsilon^{\prime}=\widetilde{\nu}(\widetilde{h})+n_{l, g_{l}} \bar{\beta}_{l, g_{l}}-\beta_{l, g_{l}}$. Therefore (recall (23)), we conclude that:

(a.2) $\nu_{l}\left(J a c\left(f_{l}^{\prime}\right)\right)=\nu_{l}\left(\frac{\partial f_{l}^{\prime}}{\partial y}\right)=\widetilde{\epsilon}+\widetilde{\nu}(\widetilde{h})$. Let $\epsilon^{\prime}$ denote this integer, then:

(b.2) for all $n \geq 0$, the class of $\frac{\partial F_{l ; e \epsilon^{\prime}+n}^{\prime}}{\partial Y_{n}}$ in $\mathcal{O}_{\left(C_{l}\right)_{\infty}, P_{l}^{\prime}}$ is a unit. Besides, if $h_{l}:=\frac{\partial f_{l}^{\prime}}{\partial y}$ then the class of $\frac{\partial F_{l ; e \epsilon^{\prime}+n}^{\prime}}{\partial Y_{n}}-H_{l ; e \epsilon^{\prime}}$ in $\mathcal{O}_{\left(C_{l}\right)_{\infty}, P_{l}^{\prime}}$ belongs to $P_{l}^{\prime}$.

(c.2) for $n^{\prime}>n$, the class of $\frac{\partial F_{l ; e \epsilon+n}^{\prime}}{\partial Y_{n^{\prime}}}$ in $\mathcal{O}_{\left(C_{l}\right)_{\infty}, P_{l}^{\prime}}$ belongs to $P_{l}^{\prime} \mathcal{O}_{\left(C_{l}\right)_{\infty}, P_{l}^{\prime}}$.

(d.2) $F_{l ; 0}^{\prime}, \ldots, F_{l ; e \epsilon-1}^{\prime}$ belong to $\left(\mathcal{F}^{\prime}{ }_{l}\right)^{2}\left(k\left[\underline{v}, x_{1}, y\right]_{\infty}\right)_{H_{0}}$. 
Now, let $b$ be the smallest nonnegative integer such that $g_{l}^{\prime}:=h^{b} f_{l}^{\prime}$ belongs to $k\left[\underline{v}, x_{1}, y\right]$ and let $\left\{b_{j, r}\right\}_{(j, r) \in \mathcal{J} *}$ be a minimal sequence of nonnegative integers such that

$$
\begin{aligned}
& f_{l}\left(x_{1}, \ldots, x_{d}, y_{l}\right):= \\
& =\prod_{(j, r) \in \mathcal{J}^{*}} q_{j, r}^{b_{j, r}} g_{l}^{\prime}\left(\frac{q_{2, g_{2}+1}}{q_{1,0}^{b_{2,0}} \ldots q_{2, g_{2}}^{b_{2, g_{2}}}}, \ldots, \frac{q_{\delta, g_{\delta}+1}}{q_{\delta, 0}^{b_{\delta, 0}} \ldots q_{\delta, g_{\delta}}^{b_{\delta, g_{\delta}}}}, x_{\delta+1}, \ldots, x_{d}, x_{1}, y_{l}\right)
\end{aligned}
$$

belongs to $k\left[x_{1}, \ldots, x_{d}, y_{l}\right]$, being $y_{l}$ an indeterminacy (recall (16) and (18)). Therefore we have

$$
f_{l}\left(x_{1}, \ldots, x_{d}, x_{l}\right)=0 .
$$

From (9) and (a.2) it follows that

$$
\epsilon_{l}:=\nu\left(J a c\left(f_{l}\right)\right)=\nu\left(\frac{\partial f_{l}}{\partial y_{l}}\right)=\nu\left(\prod_{(j, r) \in \mathcal{J}^{*}} q_{j, r}^{b_{j, r}} h^{b}\right)+\epsilon^{\prime}
$$

i.e. (i) in the statement of the proposition holds. From (b.2) and (c.2) we obtain that (ii) also holds.

For $0 \leq s \leq g_{l}+1$, let $b(l, s)$ be the smallest nonnegative integer such that $g_{l, s}^{\prime}:=$ $h^{b(l, s)} f_{l, s}^{\prime}$ belongs to $k\left[\underline{v}, x_{1}, y\right]$ and let $\left\{b_{j, r}(l, s)\right\}_{(j, r) \in \mathcal{J}^{*}}$ be a minimal sequence of nonnegative integers such that

$$
\begin{aligned}
& z_{l, s}:=
\end{aligned}
$$

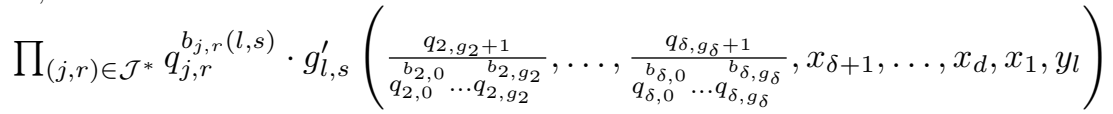

belongs to $k\left[x_{1}, \ldots, x_{d}, y_{l}\right]$. Set $\bar{\alpha}_{l, s}:=\nu\left(x_{l, s}\right)$, being $x_{l, s}$ the class of $z_{l, s}$ in $\mathcal{O}_{X^{\prime}}$, and $\mathcal{Z}_{l}:=\left\{Z_{l, s ; n}\right\}_{1 \leq s \leq g_{l}, 0 \leq n<e \bar{\alpha}_{l, s}}$. Then, from (d.2) and applying also the second assertion in (22), we conclude that

$$
F_{l ; 0}, \ldots, F_{l ; e \epsilon_{l}-1} \in\left(\mathcal{Q} \cup \mathcal{Z}_{l}\right)^{2}\left(\prod_{(j, r) \in \mathcal{J}^{*}} \bar{T}_{j, r}^{-1} k\left[x_{1}, \ldots, x_{d}, y_{l}\right]_{\infty}\right)_{\bar{H}_{0}}
$$

where, if we consider $h$ as an element of $k\left(x_{1}, \ldots, x_{d}\right)$, i.e. replace $v_{j}$ by $\frac{q_{j, g_{j}+1}}{q_{j, 0}^{b_{j, 0} \ldots q_{j, g_{j}}}}$ (resp. $x_{j}$ ), for $1 \leq j<\delta$ (resp. $\delta+1 \leq j \leq d$ ), then $\bar{H}_{0} \in \prod_{(j, r) \in \mathcal{J}^{*}} \bar{T}_{j, r}^{-1} k\left[x_{1}, \ldots, x_{d}\right]_{\infty}$ satisfies $H_{0} \equiv \bar{H}_{0} \bmod P_{e E}^{Y}$, as in 3.5. In particular, if $L:=\bar{H}_{0} \cdot \prod_{(j, r) \in \mathcal{J}^{*}} \bar{Q}_{j, r ; e \bar{\beta}_{j, r}}$, we obtain that $F_{l ; 0}, \ldots, F_{l ; e \epsilon_{l}-1} \in\left(\mathcal{Q} \cup \mathcal{Z}_{l}\right)^{2}\left(k\left[x_{1}, \ldots, x_{d}, y_{l}\right]_{\infty}\right)_{L}$. Setting $G_{l}=$ $L \cdot \prod_{s=1}^{g_{l}} Z_{l, s ; e \bar{\alpha}_{l, s}}$, and applying $(25)$ and that $\mathcal{Q}$ is a regular system of parameters of $\mathcal{O}_{\left(\mathbb{A}^{d}\right)_{\infty}, P_{e E}^{A d}}$, we have that $\left(\mathcal{Q} \cup \mathcal{Z}_{l}\right)\left(k\left[x_{1}, \ldots, x_{d}, y_{l}\right]_{\infty}\right)_{G_{l}}$ is a prime ideal.

Finally, applying (27) we conclude that

$$
X^{\prime}=\operatorname{Spec} k\left[x_{1}, \ldots, x_{d}, y_{d+1}, \ldots, y_{N}\right] /\left(f_{d+1}, \ldots, f_{N}\right)
$$

is a $d$-dimensional complete intersection scheme in $\mathbb{A}_{k}^{N}$ containing $X_{0}$ and satisfying (i) to (iii) in (b). Besides, if we set $G=L \cdot \prod_{l=d+1}^{N} \prod_{s=1}^{g_{l}} z_{l, s ; \bar{\alpha}_{l, s}}$ then we conclude that $\left(\mathcal{Q} \cup \mathcal{Z}_{l}\right)\left(k\left[x_{1}, \ldots, x_{d}, y_{d+1}, \ldots, y_{N}\right]_{\infty}\right)_{G}$ is a prime ideal such that

$$
\left(\mathcal{Q} \cup \mathcal{Z}_{l}\right) \mathcal{O}_{X_{\infty}^{\prime}, P_{e E}^{X^{\prime}}}=P_{e E}^{X^{\prime}} \mathcal{O}_{X_{\infty}^{\prime}, P_{e E}^{X^{\prime}}}
$$

Thus, the the proposition is proved. 
Remark 3.7. Keep the notation in prop. 3.6, and fix $l, d+1 \leq l \leq N$. Denote $\underline{Y}_{n}^{(l)}:=\left(Y_{1 ; n}, \ldots, Y_{d ; n}, Y_{l ; n}\right), n \geq 0$, and $f_{l, j}^{\prime}:=\frac{\partial f_{l}}{\partial y_{j}}, j \in\{1, \ldots, d, \bar{l}\}$. Then, applying Taylor's formula it follows that, for $n \geq e \epsilon_{l}$,

$$
F_{l ; n+e \epsilon_{l}+1}=H_{l ; n+e \epsilon_{l}+1}+\sum_{j=1}^{d} \sum_{i=0}^{e \epsilon_{l}} F_{l, j ; i}^{\prime} Y_{j ; n+e \epsilon_{l}+1-i}+\sum_{i=0}^{e \epsilon_{l}} F_{l, l ; i}^{\prime} Y_{l ; n+e \epsilon_{l}+1-i}
$$

where $H_{l ; n+e+1} \in k\left[\underline{Y}_{0}^{(l)}, \ldots, \underline{Y}_{n}^{(l)}\right]$ is the coefficient in $t^{n+e \epsilon_{l}+1}$ of $f_{l}\left(\sum_{i=0}^{n} \underline{Y}_{i}^{(l)} t^{i}\right)$ (see [Re1] proof of lemma 3.2). In particular, since $\epsilon_{l}:=\nu\left(J a c\left(f_{l}\right)\right)=\nu\left(\frac{\partial f_{l}}{\partial y_{l}}\right)$, it follows that, for $n \geq e \epsilon_{l}$,

$$
\begin{aligned}
& \frac{\partial F_{l ; n+e \epsilon_{l}+1}}{Y_{l ; n+1}}=F_{l, l ; e \epsilon_{l}}^{\prime} \notin P_{e E} \\
& \frac{\partial F_{l ; n+e \epsilon_{l}+1}}{Y_{l ; n^{\prime}+1}}= \begin{cases}F_{l, l ; e \epsilon_{l}-\left(n^{\prime}-n\right)}^{\prime} \in P_{e E} & \text { for } n+1 \leq n^{\prime} \leq n+e \epsilon_{l} \\
0 & \text { for } n+e \epsilon_{l}<n^{\prime} .\end{cases}
\end{aligned}
$$

This idea, generalized to complete intersection schemes (see[Re2], proof of lemma 4.2 ) is a key point in the proof of [Re2], th. 4.1 (see 2.3 (vii) and (viii)). Proposition 3.6 is an improvement of the previous assertion to a similar property for $0 \leq n \leq e \epsilon_{l}$.

Theorem 3.8. Assume that char $k=0$. Let $X$ be a reduced separated $k$-scheme of finite type, let $\nu=\nu_{E}$ be a divisorial valuation on an irreducible component $X_{0}$ of $X$, and let $e \in \mathbb{N}$. Then

$$
\operatorname{embdim} \mathcal{O}_{\left(X_{\infty}\right)_{\text {red }}, P_{e E}}=\operatorname{embdim} \mathcal{O}_{\left(X_{\infty}\right)_{\text {red }}, P_{e E}}=e\left(\widehat{k}_{E}+1\right) .
$$

where $\widehat{k}_{E}$ is the Mather discrepancy of $X$ with respect to $E$.

Moreover, if $\rho: X \rightarrow \mathbb{A}_{k}^{d}$, where $d=\operatorname{dim} X_{0}$, is a general projection, more precisely a projection that satisfies (9), and $\mathcal{Q}=\left\{\bar{Q}_{j, r ; n}\right\}_{(j, r) \in \mathcal{J}, \quad e n_{j, r-1} \bar{\beta}_{j, r-1} \leq n \leq e \bar{\beta}_{j, r}-1}$ is a regular system of parameters of $\mathcal{O}_{\left(\mathbb{A}_{k}^{d}\right)_{\infty}, P_{e E}^{\mathbb{A} d}}$, then $\mathcal{Q}$ is a minimal system of coordinates of $\left(\left(X_{\infty}\right)_{r e d}, P_{e E}^{X}\right)$, that is, we have $\sharp \mathcal{Q}=e\left(\widehat{k}_{E}+1\right)$ and

$$
P_{e E}^{X} \mathcal{O}_{\left(X_{\infty}\right)_{r e d}, P_{e E}^{X}}=(\mathcal{Q}) \mathcal{O}_{\left(X_{\infty}\right)_{r e d}, P_{e E}^{X}}
$$

Proof. First recall that, since $\mathcal{Q}$ is a regular system of parameters of $\mathcal{O}_{\left(\mathbb{A}^{d}\right)_{\infty}, P_{e E}^{\mathbb{A}^{d}}}$ ([Re4], theorem 4.8) and $\rho: X \rightarrow \mathbb{A}_{k}^{d}$ is a dominant morphism, we have

$$
P_{e E}^{X} \widehat{\mathcal{O}_{X_{\infty}, P_{e E}^{X}}^{X}}=(\mathcal{Q}) \widehat{\mathcal{O}_{X_{\infty}, P_{e E}^{X}}}
$$

([Re3], prop. 4.5, see (xi)). From this and Nakayama's lemma, the second assertion of the theorem follows (see also (20)). Therefore, we only have to prove (31), or equivalently, the independence of the elements of $\mathcal{Q}$ in $P_{e E}^{X} /\left(P_{e E}^{X}\right)^{2}$.

Let $\tilde{X} \rightarrow X$ be an étale morphism such that each irreducible component of $\widetilde{X}$ is analytically irreducible. Let $\widetilde{X}_{0}$ be an irreducible component of $\widetilde{X}$ whose image is $X_{0}$ and let $\widetilde{\nu}$ be a divisorial valuation on $\widetilde{X}_{0}$ extending $\nu$. More precisely, if $Y \rightarrow X$ is a resolution of singularities of $X$ and $E$ is a divisor on $Y$ such that $\nu=\nu_{E}$ then $\widetilde{Y}:=Y \otimes_{X} \widetilde{X} \rightarrow \widetilde{X}$ is a resolution of singularities of $\widetilde{X}$ and we may choose a divisor $\widetilde{E}$ on $\widetilde{Y}$ whose image on $\widetilde{Y}$ is $E$, and take $\widetilde{\nu}=\nu_{\widetilde{E}}$. Then $\mathcal{O}_{\widetilde{X}_{\infty}, P_{e} \widetilde{E}}$ is étale over $\mathcal{O}_{X_{\infty}, P_{e E}}$ and, since $\Omega_{\widetilde{X} / X}=0$ we have $\widehat{k}_{\widetilde{E}}(\widetilde{X})=\widehat{k}_{E}(X)$. Therefore, it suffices to prove the theorem for $\widetilde{X}$, equivalently, we may suppose that $X_{0}$ is analytically irreducible. 
So, let us assume that $X_{0}$ is analytically irreducible. Then, we can apply prop. 3.6. Let $X^{\prime}$ be the $d$-dimensional complete intersection scheme containing $X_{0}$ defined in 3.6 and keep the notation in prop. 3.6. We have $\mathcal{O}_{\left(X_{\infty}\right)_{\text {red }}, P_{e E}} \cong$ $\mathcal{O}_{\left(X_{\infty}^{\prime}\right)_{\text {red }}, P_{e E}^{X^{\prime}}}$ and $\widehat{\mathcal{O}}_{\left(X_{\infty}\right), P_{e E}} \cong \mathcal{O}_{\left(X_{\infty}^{\prime}\right), P_{e E}^{X^{\prime}}}$ (see (ii) and (ix)). Therefore, in order to prove $(31)$ we may suppose that $X=X^{\prime}$. We will next describe the ring $\widehat{\mathcal{O}_{X_{\infty}, P_{e E}}}$, where $X=X^{\prime}$ and $P_{e E}=P_{e E}^{X}$. We will follow the ideas in example 2.7 (or corol. 4.6 in [Re3]), where an analogous description is made.

The residue field of $P_{e E}^{\mathbb{A}^{d}}$ is

$$
\kappa\left(P_{e E}^{\mathbb{A}^{d}}\right) \cong k\left(\left\{X_{1 ; n}\right\}_{n>e m_{1}} \cup\left\{X_{j ; n}\right\}_{\substack{2 \leq j \leq d \\ n \geq e m_{j}}}\right)\left[\left\{W_{j, r}\right\}_{(j, r) \in \mathcal{J}^{*}}\right] / J
$$

where we set $m_{j}:=0$ for $\delta+1 \leq j \leq d$ (see $\left.(11)\right), W_{j, r}$ is the class of $\bar{Q}_{r, j ; e} \bar{\beta}_{j, r}$, and $J$ is the ideal generated by

$$
P_{j, r, 1}\left(\frac{\bar{\mu}_{j, r, 1}\left(W_{j, r}\right)^{n_{j, r}}}{W_{1,0}^{b_{j, 0}} \cdots W_{j, r-1 ;}^{b_{j, r}-1}}, \frac{W_{2, g_{2}+1}}{W_{1,0}^{b_{2,0}} \cdots W_{2, g_{2}}^{b_{2, g_{2}}}}, \ldots, \frac{W_{j-1, g_{j-1}+1}}{W_{1,0}^{b_{j-1,0}} \cdots W_{j-1, g_{j-1}}^{b_{j-1, g_{j}-1}}}\right)
$$

(recall 3.3 and 3.4). From the property (14) satisfied by $P_{j, r, 1}$ and Hensel's lemma, it follows that we can define an embedding $\kappa\left(\mathbb{A}_{k}^{d}\right) \hookrightarrow \mathcal{O}_{\left(\mathbb{A}^{d}\right)_{\infty}, P_{e E}^{\mathbb{A}^{d}}}$ sending $X_{j ; n}$ to $X_{j ; n} \in \mathcal{O}_{\left(\mathbb{A}^{d}\right)_{\infty}, P_{e E}^{\mathbb{A}^{d}}}$, for $j=1, n>e m_{1}$, and $2 \leq j \leq d, n \geq e m_{j}$, sending $W_{1,0}$ to $X_{1 ; e m_{1}}$ and, recursively, for $(j, r) \in \mathcal{J}^{*} \backslash\{(1,0)\}$, sending $W_{j, r}$ to a root of the polynomial obtained from (32) by replacing $W_{j^{\prime}, r^{\prime}},\left(j^{\prime}, r^{\prime}\right)<(j, r)$, by its image in $\mathcal{O}_{\left(\mathbb{A}^{d}\right)_{\infty}, P_{e E}^{A^{d}}}$; this root exists by Hensel's lemma. Then we have

$$
\mathcal{O}_{\left(\mathbb{A}^{d}\right)_{\infty}, P_{e E}^{\mathbb{A}^{d}}} \cong \kappa\left(P_{e E}^{\mathbb{A}^{d}}\right)\left[\left[\left\{X_{j, r ; n}\right\}_{e n_{j, r-1} \bar{\beta}_{j, r-1}^{(j, r) \in \mathcal{J}} \leq n<e \bar{\beta}_{j, r}}\right]\right]
$$

where the image of $X_{j, r ; n}$ in $\widehat{\mathcal{O}_{\left(\mathbb{A}^{d}\right)_{\infty}, P_{e E}^{\mathbb{A}^{d}}}}$ is $\bar{Q}_{r, j ; n}$. Besides $\widehat{\mathcal{O}_{X_{\infty}, P_{e E}^{X}}^{X}}$ is a quotient of

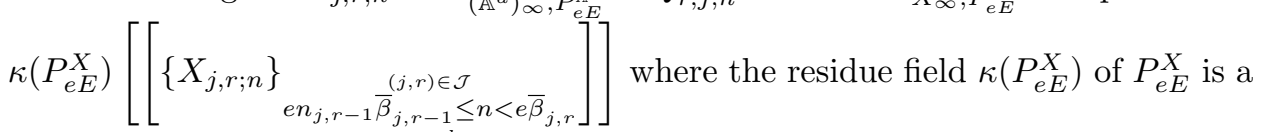
finite field extension of $\kappa\left(P_{e E}^{\mathbb{A}^{d}}\right)$.

Now, fix $l, d+1 \leq l \leq N$. Arguing analogously we obtain that

$$
\kappa_{l}:=\kappa\left(P_{e E}^{\mathbb{A}^{d}}\right)\left[\left\{W_{l, s}\right\}_{s=1}^{g_{l}}\right] / J_{l} \hookrightarrow \kappa\left(P_{e E}^{X}\right) .
$$

where $W_{l, s}$ is the class of $Z_{l, s ; e \bar{\alpha}_{l, s}}$ and $J_{l}$ is the ideal generated by the relations on $\left\{W_{l, s}\right\}_{s=1}^{g_{l}}$ induced by $G_{l, s ; \nu\left(f_{l, s}^{\prime}\right)-\left(\bar{\beta}_{l, s}-n_{l, s-1} \bar{\beta}_{l, s-1}\right)}^{\prime}, 2 \leq s \leq g_{l}$ (see (29)). Applying recursively Hensel's lemma to these relations we can define an embedding $\kappa_{l} \hookrightarrow \mathcal{O}_{X_{\infty}, P_{e E}^{X}}$ sending $X_{j ; n}$ to $X_{j ; n} \in \widehat{\mathcal{O}_{X_{\infty}, P_{e E}^{X}}}$, for $j=1, n>e m_{1}$, and $2 \leq j \leq d, n \geq e m_{j}$, and sending $W_{1,0}$ to $X_{1 ; e m_{1}} \in \widehat{\mathcal{O}_{X_{\infty}, P_{e E}^{X}}}$. In particular, for each $n \geq 0$ we have defined $Y_{l ; n}^{(0)} \in \kappa_{l}$ such that $Y_{l ; n}-Y_{l ; n}^{(0)} \in\left(\mathcal{Q} \cup \mathcal{Z}_{l}\right)$. Arguing recursively on $m \geq 1$ and $n \geq 0$, with the lexicographic order on $(m, n)$, from $\left\{F_{l ; e \epsilon_{l}+n}\right\}_{n \geq 0}$, applying property (ii) in prop. 3.6 (b) and Hensel's lemma, and reasoning as in corol. 5.6 in [Re3] it follows that, for $m, n \geq 0$, there exists $Y_{l ; n}^{(m)} \in \kappa_{l}\left[\left\{X_{j, r ; n}\right\}_{(j, r) \in \mathcal{J}, e n_{j, r-1} \bar{\beta}_{j, r-1} \leq n<e \bar{\beta}_{j, r}}\right]$ such that,

$$
F_{e \epsilon_{l}+n} \equiv L_{e \epsilon_{l}}^{(m, n)}\left(Y_{l ; n}-Y_{l ; n}^{(m)}\right) \quad \bmod \left(\mathcal{Q} \cup \mathcal{Z}_{l}\right)^{m}
$$

in the $\operatorname{ring}\left(k\left[x_{1}, \ldots, x_{d}, y_{l}\right]_{\infty}\right)_{\left(\mathcal{Q} \cup \mathcal{Z}_{l}\right)}$ where $L_{e \epsilon_{l}}^{(m, n)}$ is a unit. More precisely, $L_{e \epsilon_{l}}^{(m, n)}-$ $F_{l, l ; e \epsilon_{l}}^{\prime} \in\left(\mathcal{Q} \cup \mathcal{Z}_{l}\right)$ where recall that $f_{l, l}^{\prime}:=\frac{\partial f_{l}}{\partial y_{l}}$. 
Therefore, $Y_{l ; n}^{(m)}-Y_{l ; n}^{(m)} \in\left(\mathcal{Q} \cup \mathcal{Z}_{l}\right)^{m}$ by $(33)$, hence we have defined series $\widetilde{Y}_{l ; n} \in$

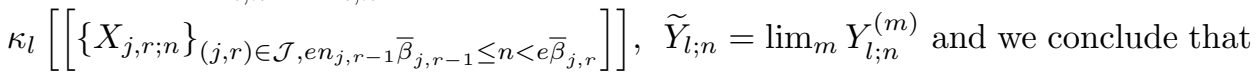

$$
\kappa\left(P_{e E}^{X}\right)=\kappa\left(P_{e E}^{Z}\right)\left[\left\{W_{l, s}\right\}_{(l, s) \in \mathcal{L}}\right] / \sum_{l=d+1}^{N} J_{l}
$$

and

$$
\widehat{\mathcal{O}_{X_{\infty}, P_{e E}^{X}}^{X}} \cong \kappa\left(P_{e E}^{X}\right)\left[\left[\left\{X_{j, r ; n}\right\}_{e n_{j, r-1} \bar{\beta}_{j, r-1} \leq n<e \bar{\beta}_{j, r}}^{(j, r) \in \mathcal{J}}\right]\right] /\left(\left\{\widetilde{F}_{l ; n}\right\}_{\substack{d+1 \leq l \leq N \\ 0 \leq n \leq e \epsilon_{l}-1}}\right)
$$

where, for $d+1 \leq l \leq N, 0 \leq n \leq e \epsilon_{l}-1, \widetilde{F}_{l ; n}$ is obtained from $F_{l ; n}$ by substituting $Y_{l ; n^{\prime}}$ by $\tilde{Y}_{l ; n}, 0 \leq n^{\prime} \leq n$ (see (25) in [Re3]). In fact, we have applied the definition $\widehat{\mathcal{O}_{X_{\infty}, P_{e E}^{X}}}:=\lim _{\leftarrow m} \mathcal{O}_{X_{\infty}, P_{e E}^{X}} /\left(P_{e E}^{X}\right)^{m+1}$ and also that $P_{e E}^{X} \mathcal{O}_{X_{\infty}, P_{e E}^{X}}=$ $(\mathcal{Q} \cup \mathcal{Z}) \mathcal{O}_{X_{\infty}, P_{e E}^{X}}$ and $\mathcal{O}_{X_{\infty}}=k\left[x_{1}, \ldots, x_{d}, y_{d+1}, \ldots y_{N}\right]_{\infty} /\left(\left\{F_{l ; n}\right\}_{d+1 \leq l \leq N, n \geq 0}\right)$. Besides, if $\widetilde{Z}_{l, s ; n}$ denotes the series obtained from $Z_{l, s ; n}$ by substituting $Y_{l ; n^{\prime}}$ by $\widetilde{Y}_{l ; n}, 0 \leq n^{\prime} \leq n$, then we have

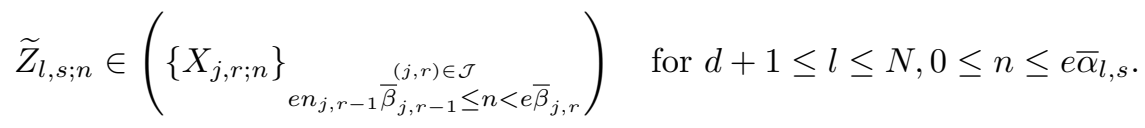

Since $F_{l ; 0}, \ldots, F_{l ; e \epsilon_{l}-1} \in\left(\mathcal{Q} \cup \mathcal{Z}_{l}\right)^{2} \kappa\left(P_{e E}^{\mathbb{A}^{d}}\right)\left[\left[\left\{X_{j, r ; n}\right\}_{(j, r) \in \mathcal{J}, e n_{j, r-1} \bar{\beta}_{j, r-1} \leq n<e \bar{\beta}_{j, r}}\right]\right]$ by (iii) in (b) in prop. 3.6, applying (35) we conclude that that

$$
\widetilde{F}_{l ; n} \in\left(\left\{X_{j, r ; n}\right\}_{e n_{j, r-1} \bar{\beta}_{j, r-1} \leq n<e \bar{\beta}_{j, r}}^{(j, r) \in \mathcal{J}}\right)^{2} \text { for } d+1 \leq l \leq N, 0 \leq n \leq ; e \epsilon_{l}-1 .
$$

Therefore, the images of $\left\{X_{j, r ; n}\right\}_{(j, r) \in \mathcal{J}, e n_{j, r-1} \bar{\beta}_{j, r-1} \leq n<e \bar{\beta}_{j, r}}$ define a basis of $P_{e E}^{X} \widehat{\mathcal{O}_{X_{\infty}, P_{e E}^{X}}} /\left(P_{e E}^{X} \widehat{\mathcal{O}_{X_{\infty}, P_{e E}^{X}}}\right)^{2}$. Thus we obtain(31), and this finishes the proof.

Remark 3.9. $\quad$ Let $X$ be a reduced separated scheme of finite type over a field $k$ of characteristic zero. Let $P$ be any stable point of $X_{\infty}$ and suppose that $X$ is nonsingular at the center $P_{0}$ of $P$ and that $P_{0}$ is not the generic point of $X$. There exists a birational and proper morphism $\pi: Y \rightarrow X$ such that the center of $\nu_{P}$ on $Y$ is a divisor $E$, and $e \in \mathbb{N}$ such that $\nu_{P}=e \nu_{E}$ ([Re3], (vii) in prop. 3.7, see $(\mathrm{v}))$. Let $P^{Y} \in Y_{\infty}$ whose image by $\pi_{\infty}$ is $P$, let $\rho: X \rightarrow \mathbb{A}_{k}^{d}$ be a general projection and let $P^{\mathbb{A}^{d}}$ be the image of $P$ in $\left(\mathbb{A}_{k}^{d}\right)_{\infty}$. Then $k_{E}\left(\mathbb{A}^{d}\right)=\widehat{k}_{E}$ where $\widehat{k}_{E}$ is the Mather discrepancy of $X$ with respect to $E$, and we have $\operatorname{dim} \mathcal{O}_{\left(\mathbb{A}^{d}\right)_{\infty}, P^{\mathbb{A}^{d}}}=$ $e \widehat{k}_{E}+\operatorname{dim} \mathcal{O}_{Y_{\infty}, P^{Y}}($ see $($ xiii $))$. Recall that $P \supseteq P_{e E}^{X}$, hence $P^{\mathbb{A}^{d}} \supseteq P_{e E}^{\mathbb{A}^{d}}$ and, if $\mathcal{Q}$ is a regular system of parameters of $\mathcal{O}_{\left(\mathbb{A}^{d}\right)_{\infty}, P_{e E}^{\mathbb{A}}}$, then $\mathcal{Q} \subset P$. Note that, since $\nu_{P}=e \nu_{E}$, the proof of prop. 3.6 extends to this case, and we obtain that the complete intersection scheme $X^{\prime}$ and the set $\mathcal{Z}$ defined in proposition 3.6 for the valuation $\nu_{E}$ and $e$ also satisfy the properties obtained replacing $P_{e E}$ by $P$ in (i) to (iii) in prop 3.6 (b). Then, from the proof of theorem 3.8 it follows that

$$
\operatorname{embdim} \mathcal{O}_{\left(X_{\infty}\right)_{\text {red }}, P}=\operatorname{embdim} \mathcal{O}_{\left(X_{\infty}\right)_{\text {red }}, P}=e\left(\widehat{k}_{E}+\operatorname{dim} \mathcal{O}_{Y_{\infty}, P^{Y}}\right) .
$$




\section{A LOWER BOUND FOR THE DIMENSION}

Recall that, given a divisorial valuation $\nu=\nu_{E}$ on $X$, the Mather-Jacobian log-discrepancy of $X$ with respect to $E$ is defined to be

$$
a_{M J}(E ; X):=\widehat{k}_{E}-\nu_{E}\left(J a c_{X}\right)+1
$$

where $J a c_{X}$ is the Jacobian ideal of $X$ (see [I]).

Theorem 4.1. Assume that char $k=0$. Let $X$ be a reduced separated $k$-scheme of finite type, let $\nu=\nu_{E}$ be a divisorial valuation on an irreducible component $X_{0}$ of $X$, and let $e \in \mathbb{N}$. Then we have

$$
\operatorname{dim} \widehat{\mathcal{O}_{X_{\infty}, P_{e E}^{X}}} \geq e a_{M J}(E ; X)
$$

In particular, if $X$ is normal and complete intersection then

$$
\operatorname{dim} \widehat{\mathcal{O}_{X_{\infty}, P_{e E}^{X}}} \geq e\left(k_{E}+1\right)
$$

Proof. It is always possible to embed $X$ in a complete intersection scheme $X^{\prime}$ such that $\widehat{k}_{E}(X)=\widehat{k}_{E}\left(X^{\prime}\right)$ and $\nu_{E}\left(J a c_{X}\right)=\nu_{E}\left(J a c_{X^{\prime}}\right)$. Hence, since $\widehat{\mathcal{O}_{\left(X_{\infty}\right), P_{e E}}} \cong$ $\mathcal{O}_{\left(X_{\infty}^{\prime}\right), P_{E}^{X^{\prime}}}$ (see (ii) and (ix)), it suffices to prove the result for $X^{\prime}$. That is, we may assume that $X$ is a complete intersection, more precisely, we may suppose that

$$
X=\operatorname{Spec} k\left[x_{1}, \ldots, x_{N}\right] /\left(f_{1}, \ldots, f_{N-d}\right) .
$$

We may also suppose that (9) holds, i.e.

$$
\operatorname{ord}_{E} \pi^{*}\left(d x_{1} \wedge \ldots \wedge d x_{d}\right)=\widehat{k}_{E}
$$

For simplicity in the notation we will prove the result when $e=1$; the proof when $e>1$ follows in the same way. Let $\rho: X \rightarrow \mathbb{A}_{k}^{d}$ be the projection on the first $d$ coordinates, let $\eta: Y \rightarrow \mathbb{A}_{k}^{d}$ be the composition $\eta=\rho \circ \pi$, let $P_{E}^{\mathbb{A}^{d}}$ be the image of $P_{E}^{Y}$ by $\eta_{\infty}$ and let $\mathcal{Q}=\left\{\bar{Q}_{j, r ; n}\right\}_{(j, r) \in \mathcal{J}, n_{j, r-1} \bar{\beta}_{j, r-1} \leq n \leq \bar{\beta}_{j, r}-1}$ be a regular system of parameters of $\mathcal{O}_{\left(\mathbb{A}^{d}\right)_{\infty}, P_{E}^{\mathbb{A}^{d}}}$ associated to $\left\{q_{j, r}\right\}_{(j, r) \in \mathcal{J}}$, as in 3.5. So we have

$$
P_{E}^{X} \mathcal{O}_{\left(X_{\infty}\right)_{\mathrm{red}}, P_{E}^{X}}=\left(\left\{\bar{Q}_{j, r ; n}\right\}_{(j, r) \in \mathcal{J}, n_{j, r-1} \bar{\beta}_{j, r-1} \leq n \leq \bar{\beta}_{j, r}-1}\right) \mathcal{O}_{\left(X_{\infty}\right)_{\mathrm{red}}, P_{E}^{X}} .
$$

(theorem 3.8).

Let us consider the following $(N-d) \times(N-d)$-matrix with coefficients in $k\left[x_{1}, \ldots, x_{N}\right]$ :

$$
\Delta:=\left(\frac{\partial f_{i}}{\partial x_{d+j}}\right)_{1 \leq i, j \leq N-d}
$$

and let $d_{j_{1}, \ldots, j_{r}}^{i_{1}, \ldots, i_{r}}$ denote the determinant of the $r \times r$ - minor of $\Delta$ defined by the rows $i_{1}, \ldots, i_{r}$ and the columns $j_{1}, \ldots, j_{r}$. After reordering $\left\{x_{d+j}\right\}_{j=1}^{N-d}$ we may suppose that

$$
\nu_{E}\left(d_{1, \ldots, i}^{1, \ldots, i}\right)=\inf \left\{\nu_{E}\left(d_{1, \ldots, i-1, j}^{1, \ldots, i-1, i}\right)\right\}_{j=i}^{N-d} \text { for } 1 \leq i \leq N-d .
$$

For $1 \leq i \leq N-d$ set

$$
\delta_{i}:=\nu_{E}\left(d_{1, \ldots, i}^{1, \ldots, i}\right) \quad \epsilon_{i}:=\inf \left\{\nu_{E}\left(\frac{\partial f_{i}}{\partial x_{d+j}}\right)\right\}_{j=1}^{N-d}=\inf \left\{\nu_{E}\left(d_{j}^{i}\right)\right\}_{j=1}^{N-d}
$$

and note that $\delta_{1}=\epsilon_{1}$ and $\delta_{N-d}:=\nu_{E}\left(J a c_{X}\right)$ by (9). It can be proved by recurrence that, for $1 \leq l \leq N-d, l \leq i, j \leq N-d$, we have

$$
d_{1, \ldots, l-1, j}^{1, \ldots, l-1, i} \cdot d_{1, \ldots, l-2}^{1, \ldots, l-2}=d_{1, \ldots, l-2, j}^{1, \ldots, l-2, i} \cdot d_{1, \ldots, l-1}^{1, \ldots, l-1}-d_{1, \ldots, l-2, l-1}^{1, \ldots, l-2, i} \cdot d_{1, \ldots, l-2, j}^{1, \ldots, l-2, l-1} .
$$


Let $f_{1, i}^{\prime}:=\frac{\partial f_{1}}{\partial x_{i}}, 1 \leq i \leq N$, thus $f_{1, d+i}^{\prime}=d_{i}^{1}, 1 \leq i \leq N-d$. Let $\sum_{n \geq 0} F_{1, i ; n}^{\prime} t^{n}$ (resp. $\left.\sum_{n>0} D_{j_{1}, \ldots, j_{r} ; n}^{i_{1}, \ldots, i_{r}} t^{n}\right)$ denote the image of $f_{1, i}^{\prime}\left(\operatorname{resp} . d_{j_{1}, \ldots, j_{r}}^{i_{1}, \ldots, i_{r}}\right)$ in $k\left[x_{1}, \ldots, x_{N}\right]_{\infty}$. Given $a_{1}>\epsilon_{1}$ and $n>\left(a_{1}-\epsilon_{1}\right)$, applying Taylor's formula to $f_{1}\left(w_{0}+t^{n-\left(a_{1}-\epsilon_{1}\right)} w_{1}\right)$, where $w_{0}=\sum_{i=0}^{n-\left(a_{1}-\epsilon_{1}\right)-1} \underline{x}_{i} t^{i}$ and $w_{1}=\sum_{i \geq n-\left(a_{1}-\epsilon_{1}\right)} \underline{x}_{i} t^{i-\left(n-\left(a_{1}-\epsilon_{1}\right)\right)}$, we obtain that for $n>n_{1}:=2 a_{1}-\epsilon_{1}$ (i.e. $\left.2\left(n-\left(a_{1}-\epsilon_{1}\right)\right)>n+\epsilon_{1}\right)$ we have

$$
F_{1 ; \epsilon_{1}+n}=H_{1 ; n}^{\prime}\left(\underline{X}_{0}, \ldots, \underline{X}_{n-\left(a_{1}-\epsilon_{1}\right)-1}\right)+\sum_{i=1}^{N} \sum_{r=0}^{a_{1}} F_{1, i ; r}^{\prime} X_{i ; n+\epsilon_{1}-r}
$$

where $H_{1, n}^{\prime} \in k\left[\underline{X}_{0}, \ldots, \underline{X}_{n-\left(a_{1}-\epsilon_{1}\right)-1}\right]$ (see [Re2], proof of theorem 4.1, or equality (30) in remark 3.7, where the same argument is applied). Hence, there exists a polynomial $H_{1 ; n} \in k\left[\underline{X}_{0}, \ldots, \underline{X}_{n-\left(a_{1}-\epsilon_{1}\right)-1},\left\{X_{j ; n^{\prime}}\right\}_{\substack{1 \leq j \leq d \\ n-\left(a_{1}-\epsilon_{1}\right) \leq n^{\prime} \leq n+\epsilon_{1}}}\right]$ such that

$$
\begin{aligned}
F_{1 ; \epsilon_{1}+n}= & H_{1 ; n}\left(\underline{X}_{0}, \ldots, \underline{X}_{n-\left(a_{1}-\epsilon_{1}\right)-1},\left\{X_{j ; n^{\prime}}\right\}_{\substack{1 \leq j \leq d \\
n^{\prime} \leq n+\epsilon_{1}}}\right)+ \\
& +\sum_{i=1}^{N-d} \sum_{r=\epsilon_{1}}^{a_{1}} D_{i ; r}^{1} X_{d+i ; n+\epsilon_{1}-r} \bmod \left(\left\{D_{i ; s}^{1}\right\}_{\substack{1 \leq i \leq N-d \\
0 \leq s<\epsilon_{1}}}\right) .
\end{aligned}
$$

It follows that, for $n>n_{1}$ there exists

$$
X_{d+1 ; n}^{(1)} \in k\left[\left\{X_{j ; n^{\prime}}\right\}_{\substack{1 \leq j \leq d \\ 0 \leq n^{\prime} \leq n+\epsilon_{1}}} \cup\left\{X_{d+1 ; n^{\prime}}\right\}_{0 \leq n^{\prime} \leq n_{1}} \cup\left\{X_{d+i ; n^{\prime}}\right\}_{\substack{2 \leq i \leq N-d \\ 0 \leq n^{\prime} \leq n}}\right]_{D_{1 ; \epsilon_{1}}^{1}}
$$

such that

$$
F_{1 ; \epsilon_{1}+n}=D_{1 ; \epsilon_{1}}^{1}\left(X_{d+1 ; n}-X_{d+1 ; n}^{(1)}\right) \quad \bmod \left(\left\{D_{i ; s}^{1}\right\}_{\substack{1 \leq i \leq N-d \\ 0 \leq s<\epsilon_{1}}} \cup\left\{F_{1 ; \epsilon_{1}+n^{\prime}}\right\}_{n_{1}<n^{\prime}<n}\right)
$$

in the ring $\left(k\left[x_{1}, \ldots, x_{N}\right]_{\infty}\right)_{D_{1 ; \epsilon_{1}}^{1}}$. Besides, it can be proved by recurrence that, for $n>n_{1}+a_{1}-\epsilon_{1}, 2 \leq i \leq N-d$ and $0 \leq r \leq a_{1}-\epsilon_{1}$ we have

$$
\frac{\partial X_{d+1 ; n}^{(1)}}{\partial X_{d+i ; n-r}}=-\sum_{s=0}^{r} \frac{D_{i ; \epsilon_{1}+s}^{1}}{D_{1 ; \epsilon_{1}}^{1}} B_{r-s}^{1} \quad \bmod \left(\left\{D_{i ; s}^{1}\right\}_{\substack{1 \leq i \leq N-d \\ 0 \leq s<\epsilon_{1}}}\right) .
$$

where

$$
B_{r-s}^{1}:=\sum_{k_{1}, \ldots, k_{m}, b_{1}, \ldots, b_{m}}(-1)^{b} \frac{b !}{b_{1} ! \cdots b_{m} !} \frac{\left(D_{1 ; \epsilon_{1}+k_{1}}^{1}\right)^{b_{1}} \cdots\left(D_{1 ; \epsilon_{1}+k_{m}}^{1}\right)^{b_{m}}}{\left(D_{1 ; \epsilon_{1}}^{1}\right)^{b}} .
$$

with $k_{1}, \ldots, k_{m}, b_{1}, \ldots, b_{m}$ runnig over all positive integers satisfying $k_{1}<k_{2}<$ $\ldots<k_{m}$ and $\sum_{i=1}^{m} b_{i} k_{i}=r-s$, and $b:=\sum_{i=1}^{m} b_{i}$.

Analogously, taking $a_{2}>\epsilon_{2}$, applying Taylor's formula to $f_{2}$, and then replacing $X_{d+1 ; n^{\prime}}$ by $X_{d+1 ; n^{\prime}}^{(1)}$ for $n^{\prime}>n_{1}$, i.e. considering the image $F_{2 ; \epsilon_{2}+n}^{(1)}$ of $F_{2 ; \epsilon_{2}+n}$ in $k\left[\left\{X_{j ; n^{\prime}}\right\}_{\substack{1 \leq j \leq d \\ 0 \leq n^{\prime} \leq \epsilon_{2}+n}} \cup\left\{X_{d+1 ; n^{\prime}}\right\}_{0 \leq n^{\prime} \leq n_{1}} \cup\left\{X_{d+i ; n^{\prime}}\right\}_{\substack{2 \leq i \leq N-d \\ 0 \leq n^{\prime} \leq n}}\right]_{D_{1 ; \epsilon_{1}}^{1}}$, we obtain that for $n>>0,2 \leq i \leq N-d, 0 \leq r \leq \inf \left\{\left(a_{1}-\epsilon_{1}\right),\left(a_{2}-\epsilon_{2}\right)\right\}$, we have

(41) $\frac{\partial F_{2 ; \epsilon_{2}+n}^{(1)}}{\partial X_{d+i ; n-r}}=\sum_{s=0}^{r} \frac{D_{1, i ; \epsilon_{1}+\epsilon_{2}+s}^{1,2}}{D_{1 ; \epsilon_{1}}^{1}} B_{r-s}^{1} \bmod \left(\left\{D_{i ; s}^{1}\right\}_{\substack{1 \leq i \leq N-d \\ 0 \leq s<\epsilon_{1}}} \cup\left\{D_{i ; s}^{2}\right\}_{\substack{1 \leq i \leq N-d \\ 0 \leq s<\epsilon_{2}}}\right)$.

In fact, to conclude (41) we have to apply Taylor's development as in (39) and also the identities (40). Hence, if $\left(a_{1}-\epsilon_{1}\right)$ and $\left(a_{2}-\epsilon_{2}\right)$ are bigger than $\left(\delta_{2}-\delta_{1}-\epsilon_{2}\right)$, 
for $n>>0,0 \leq r \leq \inf \left\{\left(a_{1}-\epsilon_{1}\right)-\left(\delta_{2}-\delta_{1}-\epsilon_{2}\right),\left(a_{2}-\epsilon_{2}\right)-\left(\delta_{2}-\delta_{1}-\epsilon_{2}\right)\right\}$ and $2 \leq i \leq N-d$, we have

$$
\begin{aligned}
\frac{\partial F_{2 ; \delta_{2}-\delta_{1}+n}^{(1)}}{\partial X_{d+i, n-r}} & =\sum_{s=0}^{r} \frac{D_{1, i ; \delta_{2}+s}^{1,2}}{D_{1 ; \epsilon_{1}}^{1}} B_{r-s}^{1} \\
\bmod & \left(\left\{D_{i ; s}^{1}\right\}_{\substack{1 \leq i \leq N-d \\
0 \leq s<\epsilon_{1}}} \cup\left\{D_{i ; s}^{2}\right\}_{\substack{1 \leq i \leq N-d \\
0 \leq s<\epsilon_{2}}} \cup\left\{D_{1, i ; s}^{1,2}\right\}_{\substack{1 \leq i \leq N-d \\
0 \leq s<\delta_{2}}}\right) .
\end{aligned}
$$

In particular

$$
\frac{\partial F_{2 ; \delta_{2}-\delta_{1}+n}^{(1)}}{\partial X_{d+i, n}} \equiv \frac{D_{1, i, \delta_{2}}^{1,2}}{D_{1 ; \epsilon_{1}}^{1}} \text { and } \quad \frac{\partial F_{2 ; \delta_{2}-\delta_{1}+n}^{(1)}}{\partial X_{d+i, n^{\prime}}} \equiv 0 \text { for } n^{\prime}>n .
$$

This implies that there exists $n_{2}$ such that for $n>n_{2}$ there exists

$$
X_{d+2 ; n}^{(1)} \in k\left[\left\{X_{j ; n^{\prime}}\right\}_{n^{\prime} \leq n+j \delta_{2}-\delta_{1}}^{1 \leq j} \cup\left\{X_{d+i ; n^{\prime}}\right\}_{\substack{1 \leq i \leq 2 \\ n^{\prime} \leq n_{i}}} \cup\left\{X_{d+i ; n^{\prime}}\right\}_{\substack{3 \leq i \leq N-d \\ n^{\prime} \leq n}}\right]_{D_{1 ; \epsilon_{1}}^{1} \cdot D_{1,2 ; \delta_{2}}^{1,2}}
$$

such that

$$
\begin{aligned}
& F_{2, \delta_{2}-\delta_{1}+n}=\frac{D_{1,2 ; \delta_{2}}^{1,2}}{D_{1 ; \epsilon_{1}}^{1}}\left(X_{d+2 ; n}-X_{d+2 ; n}^{(1)}\right) \\
& \bmod \left(\left\{D_{i ; s_{1}}^{j}, D_{1, i ; s_{2}}^{1,2}\right\}_{\substack{1 \leq i \leq N-d \\
1 \leq j \leq 2 \\
s_{1}<\delta_{1}, s_{2}<\delta_{2}}} \cup\left\{F_{1 ; \epsilon_{1}+n^{\prime}}\right\}_{n^{\prime}=n_{1}+1}^{n+\left(\delta_{2}-\delta_{1}-\epsilon_{2}\right)} \cup\left\{F_{2 ; \delta_{2}-\epsilon_{1}+n^{\prime}}\right\}_{n_{2}<n^{\prime}<n}\right)
\end{aligned}
$$

in the ring $\left(k\left[x_{1}, \ldots, x_{N}\right]_{\infty}\right)_{D_{1 ; \epsilon_{1}}^{1} \cdot D_{1,2 ; \delta_{2}}^{1,2}}$ and

$$
\frac{\partial X_{d+2 ; n}^{(1)}}{\partial X_{d+i ; n-r}}=-\sum_{s=0}^{r} \frac{D_{1, i ; \delta_{2}+s}^{1,2}}{D_{1,2 ; \delta_{2}}^{1,2}} B_{r-s}^{2} \bmod \left(\left\{D_{i ; s}^{j}\right\}_{\substack{1 \leq i \leq N-d \\ 1 \leq j \leq 2 \\ 0 \leq s<\epsilon_{1}}} \cup\left\{D_{1, i ; s}^{1,2}\right\}_{\substack{1 \leq i \leq N-d \\ 0 \leq s<\delta_{2}}}\right)
$$

for $2 \leq i \leq N-d$ and $0 \leq r \leq \inf \left\{\left(a_{l}-\epsilon_{l}\right)-\left(\delta_{l}-\delta_{l-1}-\epsilon_{l}\right)-\ldots-\left(\delta_{2}-\delta_{1}-\epsilon_{2}\right)\right\}_{1 \leq l \leq 2}$, where we set $\delta_{0}:=0$.

Now let

$$
\mathcal{D}:=\left\{D_{i ; s}^{j}\right\}_{\substack{\leq i, j \leq N-d \\ 0 \leq s<\epsilon_{j}}} \cup\left\{D_{1, i ; s}^{1,2}\right\}_{\substack{1 \leq i \leq N-d \\ 0 \leq s<\delta_{2}}} \cup \ldots \cup\left\{D_{1,2, \ldots, N-d-1, i ; s}^{1,2, \ldots, N-d-1, N-d}\right\}_{\substack{1 \leq i \leq N-d \\ 0 \leq s<\delta_{N-d}}}
$$

and $D_{0}:=D_{1 ; \epsilon_{1}}^{1} \cdot D_{1,2 ; \delta_{2}}^{1,2} \cdots D_{1,2, \ldots, N-d ; \delta_{N-d}}^{1,2, \ldots, N-d}$. Recall that, by (37) and since $\delta_{i}=$ $\nu_{E}\left(d_{1, \ldots, i}^{1, \ldots, i}\right)$, we have that, for each element in $\mathcal{D}$, its class in $\mathcal{O}_{X_{\infty}, P_{E}^{X}}$ is in $P_{E}^{X}$ and also that the class of $D_{0}$ is a unit in $\mathcal{O}_{X_{\infty}, P_{E}^{X}}$. Following as before, we obtain that, for $1 \leq i \leq N-d$, given $a_{i}>\epsilon_{i}$, there exists $n_{i}$ such that for $n>n_{i}$ there exists

$$
X_{d+i ; n}^{(1)} \in k\left[\left\{X_{j ; n^{\prime}}\right\}_{\substack{0 \leq n^{\prime} \leq j \leq d \\ 0 \leq n+\delta_{i}-\delta_{i-1}}} \cup\left\{X_{d+j ; n^{\prime}}\right\}_{\substack{1 \leq j \leq i \\ 0 \leq n^{\prime} \leq n_{j}}} \cup\left\{X_{d+j ; n^{\prime}}\right\}_{\substack{i+1 \leq j \leq N-d \\ 0 \leq n^{\prime} \leq n}}\right]_{D_{0}}
$$

satisfying

$$
\begin{gathered}
F_{i ; \delta_{i}-\delta_{i-1}+n}=\frac{D_{1, \ldots, i ; \delta_{i}}^{1, \ldots, i}}{D_{1, \ldots, i-1 ; \delta_{i-1}}^{1, \ldots, i-1}}\left(X_{d+i ; n}-X_{d+i ; n}^{(1)}\right) \\
\bmod \left(\mathcal{D} \cup\left\{F_{j ; \delta_{j}-\delta_{j-1}+n^{\prime}}\right\}_{n_{j}<n^{\prime}<+n+\left(\delta_{i}-\delta_{i-1}-\epsilon_{i}\right)} \cup\left\{F_{i ; \delta_{i}-\delta_{i-1}+n^{\prime}}\right\}_{n_{i}<n^{\prime}<n}\right)
\end{gathered}
$$

in the ring $\left(k\left[x_{1}, \ldots, x_{N}\right]_{\infty}\right)_{D_{0}}$, and

$$
\frac{\partial X_{d+i ; n}^{(1)}}{\partial X_{d+j ; n-r}}=-\sum_{s=0}^{r} \frac{D_{1, \ldots, i-1, j ; \delta_{i}+s}^{1, \ldots, i-1, i}}{D_{1, \ldots, i ; \delta_{i}}^{1, \ldots, i}} B_{r-s}^{i} \quad \bmod (\mathcal{D})
$$


for $i \leq j \leq N-d$ and $r \leq \inf \left\{\left(a_{l}-\epsilon_{l}\right)-\left(\delta_{l}-\delta_{l-1}-\epsilon_{l}\right)-\ldots-\left(\delta_{i}-\delta_{i-1}-\epsilon_{i}\right)\right\}_{1 \leq l \leq i}$ where

$$
B_{r-s}^{i}:=\sum_{k_{1}, \ldots, k_{m}, b_{1}, \ldots, b_{m}}(-1)^{b} \frac{b !}{b_{1} ! \cdots b_{m} !} \frac{\left(D_{1, \ldots, i ; \delta_{i}+k_{1}}^{1, \ldots, i}\right)^{b_{1}} \cdots\left(D_{1, \ldots, i ; \delta_{i}+k_{m}}^{1, \ldots, i}\right)^{b_{m}}}{\left(D_{1, \ldots, i ; \delta_{i}}^{1, \ldots, i}\right)^{b}} .
$$

$k_{1}, \ldots, k_{m}, b_{1}, \ldots, b_{m}$ running over all positive integers such that $k_{1}<\ldots<k_{m}$ and $\sum_{i=1}^{m} b_{i} k_{i}=r-s$, and $b:=\sum_{i=1}^{m} b_{i}$. Note that from (43) and applying the equalities (38) it follows that for $n>>0$, the image $F_{i+1 ; \delta_{i+1}-\delta_{i}+n}^{(1)}$ of $F_{i+1 ; \delta_{i+1}-\delta_{i}+n}$ in $k\left[\left\{X_{j ; n^{\prime}}\right\}_{\substack{1 \leq j \leq d \\ 0 \leq n^{\prime} \leq \epsilon_{i+1}+n}} \cup\left\{X_{d+j ; n^{\prime}}\right\}_{\substack{1 \leq j \leq i \\ 0 \leq n^{\prime} \leq n_{j}}} \cup\left\{X_{d+j ; n^{\prime}}\right\}_{\substack{i+1 \leq j \leq N-d \\ 0 \leq n^{\prime} \leq n}}\right]_{D_{0}}$ satisfies

$$
\frac{\partial F_{i+1 ; \delta_{i+1}-\delta_{i}+n}^{(1)}}{\partial X_{d+j, n-r}}=\sum_{s=0}^{r} \frac{D_{1, \ldots, i, j ; \delta_{i}+\delta_{i+1}+s}^{1, \ldots, i, i+1}}{D_{1, \ldots, i ; \delta_{i}}^{1, \ldots, i}} B_{r-s}^{i} \bmod (\mathcal{D}) .
$$

for $i+1 \leq j \leq N-d$ and $r \leq \inf \left\{\left(a_{l}-\epsilon_{l}\right)-\left(\delta_{l}-\delta_{l-1}-\epsilon_{l}\right)-\ldots-\left(\delta_{i+1}-\delta_{i}-\epsilon_{i}\right)\right\}_{1 \leq l \leq i+1}$. This is used in the recurrence reasoning. Therefore, taking $a_{l}>\epsilon_{l}+\left(\delta_{l}-\delta_{l-1}-\right.$ $\left.\epsilon_{l}\right)+\ldots+\left(\delta_{N-d}-\delta_{N-d-1}-\epsilon_{N-d}\right)$ for $1 \leq l \leq N-d$, we conclude the existence of $n_{i}, 1 \leq i \leq N-d$, and $X_{d+i ; n}^{(1)}, 1 \leq i \leq N-d, n>n_{i}$, satisfying (42) and (43).

From the previous discussion and arguing by recurrence on $(m, i, n), m \geq 1,1 \leq$ $i \leq N-d, n \geq n_{i}+1$, with the lexicographic order, we obtain

$$
X_{d+i ; n}^{(m)} \in k\left[\left\{X_{j ; n^{\prime}}\right\}_{\substack{1 \leq j \leq d \\ n^{\prime} \geq 0}} \cup\left\{X_{d+j ; n^{\prime}}\right\}_{\substack{1 \leq j \leq N-d \\ 0 \leq n^{\prime} \leq n_{j}}}\right]_{D_{0}}
$$

satisfying

$$
\begin{gathered}
F_{i ; \delta_{i}-\delta_{i-1}+n}=\frac{D_{1, \ldots, i ; \delta_{i}}^{1, \ldots, i}}{D_{1, \ldots, i-1 ; \delta_{i-1}}^{1, \ldots, i-1}}\left(X_{d+i ; n}-X_{d+i ; n}^{(m)}\right) \\
\bmod (\mathcal{D})^{m}+\left(\left\{F_{j ; \delta_{j}-\delta_{j-1}+n^{\prime}}\right\}_{\substack{1 \leq j \leq N-d \\
n_{j}<n^{\prime}}}\right)
\end{gathered}
$$

in $\left(k\left[x_{1}, \ldots, x_{N}\right]_{\infty}\right)_{D_{0}}$. Thus we have

$$
X_{d+i ; n}^{(m+1)}-X_{d+i ; n}^{(m)} \in(\mathcal{D})^{m}+\left(\left\{F_{j ; \delta_{j}-\delta_{j-1}+n^{\prime}}\right\}_{\substack{1 \leq j \leq N-d \\ n_{j}<n^{\prime}}}\right) .
$$

Recall (36) and that the image of $\mathcal{D}$ in $\mathcal{O}_{X_{\infty}, P_{E}^{X}}$ is in $P_{E}^{X}$. Fix an embedding $\kappa\left(P_{E}^{X}\right) \hookrightarrow \widehat{\mathcal{O}_{X_{\infty}, P_{E}^{X}}}$ sending $X_{j ; n}$ to $X_{j ; n} \in \widehat{\mathcal{O}_{X_{\infty}, P_{E}^{X}}}$, for $1 \leq j \leq d, n \geq m_{j}$ (see the proof of theorem 3.8). Then, for $1 \leq i \leq N-d$ and $n>n_{i}$, the polynomials $\left\{X_{d+i ; n}^{(m)}\right\}_{m \geq 1}$ define a series

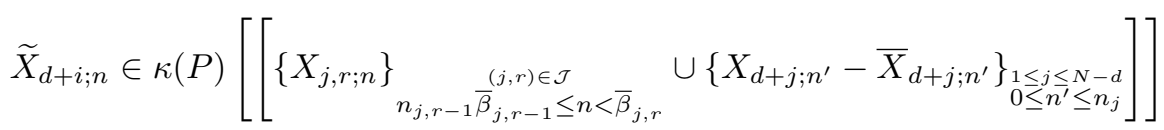

where we identify $X_{j, r ; n}$ with $\bar{Q}_{j, r ; n}$, as in the proof of th. 3.8 , and where $\bar{X}_{d+j ; n^{\prime}} \in$ $\widehat{\mathcal{O}_{X_{\infty}, P_{E}^{X}}}$ is the image of the class of $X_{d+j ; n^{\prime}}$ in $\kappa\left(P_{E}^{X}\right)$, for $1 \leq j \leq N-d, 0 \leq n^{\prime} \leq$ $n_{j}$. Setting $Y_{d+j ; n^{\prime}}:=X_{d+j ; n^{\prime}}-\bar{X}_{d+j ; n^{\prime}}, 1 \leq j \leq N-d, 0 \leq n^{\prime} \leq n_{j}$, we conclude that $\widehat{\mathcal{O}_{X_{\infty}, P_{E}^{X}}}$ is isomorphic to

$\kappa\left(P_{e E}^{X}\right)\left[\left[\left\{X_{j, r ; n}\right\}_{\substack{n_{j, r-1} \bar{\beta}_{j, r-1} \leq n<\bar{\beta}_{j, r} \\{ }^{j, r) \in \mathcal{J}}}} \cup\left\{Y_{d+j ; n^{\prime}}\right\}_{\substack{1 \leq j \leq N-d \\ n^{\prime} \leq n_{j}}}\right]\right] /\left(\left\{\widetilde{F}_{j ; n}\right\}_{\substack{1 \leq j \leq N-d \\ n \leq \delta_{j}-\bar{\delta}_{j-1}+n_{j}}}\right)$ 
where for $1 \leq j \leq N-d, 0 \leq n \leq \delta_{j}-\delta_{j-1}+n_{j}, \widetilde{F}_{j ; n}$ is obtained from $F_{j ; n}$ by substituting $X_{d+i ; n^{\prime}}$ by $\widetilde{X}_{d+i ; n^{\prime}}$, for $1 \leq i \leq N-d$ and $0 \leq n^{\prime} \leq n$, and $X_{d+j ; n^{\prime}}$ by $\bar{X}_{d+j ; n^{\prime}}+Y_{d+j ; n^{\prime}}$ for $1 \leq j \leq N-d, 0 \leq n^{\prime} \leq n_{j}$. Applying Krull's theorem we obtain that

$\operatorname{dim} \mathcal{O}_{X_{\infty}, P_{e E}^{X}} \geq \widehat{k}_{E}+1+\sum_{i=1}^{N-d}\left(n_{i}+1\right)-\sum_{i=1}^{N-d}\left(\delta_{i}-\delta_{i-1}+n_{i}+1\right)=\widehat{k}_{E}+1-\delta_{N-d}=a_{M J}(E)$.

Finally, if $X$ is normal and complete intersection, we have $a_{M J}(E)=k_{E}+1$ ([EM] appendix). Hence we conclude the result.

4.2. Recall that, given an extension of fields $k \subseteq K$, a $K$-wedge on $X$ is a $k$ morphism Spec $K[[\xi, t]] \rightarrow X$; equivalently it is a $K$-arc on $X_{\infty}$ (see (3)). Given a birational and proper $k$-morphism $p: Y \rightarrow X$ and a stable point $P$ of $X_{\infty}$, we say that $p$ satisfies the property of lifting wedges centered at $P$ if, for any field extension $K$ of the residue field $\kappa(P)$ of $P$ in $X_{\infty}$, and for any $K$-wedge $\phi:$ Spec $K[[\xi, t]] \rightarrow X$ on $X$ whose special arc is $P$ (i.e. $P$ is the image in $X_{\infty}$ of the closed point of Spec $K[[\xi]])$, there exists a $K$-wedge $\widetilde{\phi}$ : Spec $K[[\xi, t]] \rightarrow Y$ on $Y$ such that $p \circ \widetilde{\phi}=\phi$.

In [Re3], corol. 5.12, it is proved that, if $\nu=\nu_{E}$ is an essential divisorial valuation on $X$, then, the following are equivalent:

(i) $\operatorname{dim} \widehat{\mathcal{O}_{X_{\infty}, P_{E}^{X}}}=1$ and Spec $\widehat{\mathcal{O}_{X_{\infty}, P_{E}^{X}}}$ is irreducible.

(ii) $\operatorname{dim} \mathcal{O}_{X_{\infty}, P_{E}^{X}}=1$.

(iii) For every resolution of singularities $p: Y \rightarrow X, p$ satisfies the property of lifting wedges centered at $P_{E}$.

(iii') There exists a resolution of singularities $p: Y \rightarrow X$ that satisfies the condition in (iii), and such that the center of $\nu$ on $Y$ has codimension 1.

T. de Fernex and R. Docampo [dFD] have proved that, if $\nu_{E}$ is a terminal valuation then condition (iii) above holds. In fact, this follows from the proof of th.1.1 in [dFD], note that their statement in th.1.1 is weaker to condition (iii) (see [Re2], th.5.1 or [Re3] section 5). Terminal valuations are the divisorial valuations defined by the exceptional divisors of a minimal model of $X$, hence they are essential (see $[\mathrm{dFD}])$.

From this and theorem 4.1 above, corollaries 4.3 and 4.4 below follow:

Corollary 4.3. Let $X$ be a reduced separated scheme of finite type over a field $k$ of char $k=0$. Let $\nu=\nu_{E}$ be an essential divisorial valuation on an irreducible component $X_{0}$ of $X$. Consider the following conditions:

(1) $\nu_{E}$ is a terminal valuation.

(2) $\operatorname{dim} \widehat{\mathcal{O}_{X_{\infty}, P_{E}^{X}}}=1$.

(3) $a_{M J}(E ; X) \leq 1$, in particular $k_{E}(X) \leq 0$ if $X$ is normal and complete intersection.

We have that (1) implies (2) and (2) implies (3).

The following example shows that (2) does not imply (1). It has been pointed out to us by M. Mustata.

Remark 4.4. In $[\mathrm{dFD}]$, example 6.3 , the toric variety $X$ defined by the cone $\sigma$ in $\mathbb{R}^{3}$ spanned by the vectors $(1,0,0),(0,1,0)$ and $(1,1,2)$ is considered, and the divisorial valuation $\nu_{E}$ defined by $(1,1,1)$, which is not a terminal valuation. It can be proved that $\operatorname{dim} \widehat{\mathcal{O}_{X_{\infty}, P_{E}^{X}}}=1$. In this case we have $\widehat{k}_{E}(X)=2$ and $\nu_{E}\left(J a c_{X}\right)=3$, hence $a_{M J}(E ; X)=0$. 
Corollary 4.5. Let $X$ be a reduced separated scheme of finite type over a field $k$ of char $k=0$. Suppose that $X$ is normal and complete intersection. Let $\nu=\nu_{E}$ be an essential divisorial valuation on an irreducible component $X_{0}$ of $X$ and suppose that $k_{E} \geq 1$. Then, for every resolution of singularities $p: Y \rightarrow X$ such that the center of $\nu$ on $Y$ has codimension 1, $p$ does not satisfy the property of lifting wedges centered at $P_{E}$, i.e. there exist a field extension $K$ of $\kappa\left(P_{E}\right)$ and a $K$-wedge $\phi:$ Spec $K[[\xi, t]] \rightarrow X$ on $X$ whose special arc is $P_{E}$ and which does not lift to $Y$.

\section{References.}

[Bo] N. Bourbaki, Commutative Algebra, Chapter III, Springer (1989).

[dF1] T. de Fernex, Three dimensional counter-examples to the Nash problem, Compos. Math. 149 n. 9 (2013), 1519-1534.

[dF2] T. de Fernex, The space of arcs of an algebraic variety . Preprint 2016, arXiv:1604.02728.

[dFD] T. de Fernex, R. Docampo, Terminal vauations and the Nash problem, Invent. Math. 203 no. 1 (2016), 303-331.

[dFEI] T. de Fernex, L. Ein and S. Ishii, Divisorial valuations via arcs, Publ. Res. Inst. Math. Sci. 44 no. 2 (2008), 425-448.

[DL] J. Denef, F. Loeser, Germs of arcs on singular algebraic varieties and motivic integration, Invent. Math. 135 (1999), 201-232.

[ELM] L. Ein, R. Lazarsfeld, M. Mustata, Contact loci in arc spaces, Compos. Math. 140 no. 5 (2004), 1229-1244.

[EM] L. Ein and M. Mustata, Jet schemes and singularities, Proc. Symp. Pure Math. 80.2 (2009), 505-546.

[GT] R. Goldin and B. Teissier, Resolving singularities of plane analytic branches with one toric morphism. Resolution of singularities (Obergurgl, 1997), 315-340, Progr. Math., 181, Birkhauser, Basel, 2000.

[I] S. Ishii, Mather discrepancy and the arc spaces, Ann. Inst. Fourier 63, 1 (2013), 89-111.

[IK] S. Ishii, J. Kollár, The Nash problem on arc families of singularities, Duke Math. J. 120 , n.3 (2003), 601-620.

[JK] J. Johnson, J. Kollár, Arc spaces of cA-type singularities, J. Singul. 7 (2013), 238-252.

[Le] M. Lejeune-Jalabert, Courbes tracées sur un germe d'hypersurface, Amer. J. Math. 112 (1990), 525-568.

[LMR] M. Lejeune-Jalabert, H. Mourtada, A.J. Reguera, Jet schemes and minimal embedded desingularization of plane branches Revista de la Real Academia de Ciencias Exactas, Fisicas y Naturales. Serie A. Matematicas: 107, 1 (2013), 145-157.

[Mo] H. Mourtada, Jet schemes and generating sequences of divisorial valuations in dimension two, to appear in Michigan Math. J.

[Re1] A.J. Reguera, Image of the Nash map in terms of wedges, C. R. Math. Acad. Sci. Paris 338, n.5 (2004), 385-390.

[Re2] A.J. Reguera, A curve selection lemma in spaces of arcs and the image of the Nash map, Compos. Math. 142 (2006), 119-130.

[Re3] A.J. Reguera, Towards the singular locus of the space of arcs, Amer. J. Math. 131, n. 2 (2009), 313-350.

[Re4] A.J. Reguera, Coordinates at stable points of the space of arcs. Preprint 2016, < hal01305997> (https://hal.archives-ouvertes.fr/hal-01305997).

[Sp] M. Spivakovsky, Valuations in function fields of surfaces, Amer. J. Math. 112 (1990), 107-156.

[Te] B. Teissier, Overweight deformations of affine toric varieties and local uniformization, in "Valuation theory in interaction", Proceedings of the second international conference on valuation theory, Segovia-El Escorial, 2011. Edited by A. Campillo, F-V. Kuhlmann and B.Teissier. European Math. Soc. Publishing House, Congress Reports Series, Sept. $2014,474-565$

[ZT] O. Zariski with an appendix by B. Teissier, The moduli problem for plane branches, AMS 39, (2006).

H. Mourtada. Equipe Géométrie et Dynamique, Institut Mathématique de Jussieu-Paris Rive Gauche, Université Paris 7, Bâtiment Sophie Germain, case 7012, 75205 Paris Cedex 13, France. Email: hussein.mourtada@imj-prg.fr

Ana J. Reguera. Dpto. de Álgebra, Análisis Matemático, Geometría y Topología. Univ. Valladolid, 47011 Valladolid, Spain. E-mail: areguera@agt.uva.es. 